\title{
Making user-friendly experiences that leverage online collaboration
}

BY

\author{
JUAN SEBASTIAN PAZ
}

A thesis submitted to the Victoria University of Wellington in fulfilment of the requirements for the degree of Masters of Design Innovation

Victoria University of Wellington 2019 
A 90-point thesis submitted to Victoria University of Wellington in partial fulfillment of the requirements for the degree of Master of Design Innovation in Media Design

\author{
By Juan Sebastian Paz MDI \\ specialising in Media Design \\ Victoria University of Wellington School of Design 2018
}




\section{Abstract}

The recent rapid advancements in web technologies has led to an increase in the development of new devices, applications and methods of interaction that are continually evolving. However, the rate of these technological advancements outpaces those of design. This has also given rise to a new way of understanding how to work and collaborate online. Interestingly, in addition to this understanding came a corresponding increase in the frequency of online collaborations. A good example of this is a content management system. This system gives the ability for multiple users to collaborate online with the goal to maintain and create content for a website. However, during the time of writing this thesis, most content management systems come with limited functionality and not as user-friendly as they could be. As most of this systems are used by non tech-savvy users.

This thesis sets to understand social aspects of online collaboration, new design methodology, and to design and develop a user-friendly interface for both front-end and back-end admin area to leverage the online collaboration within an educational setting. Using both iterative and experimental approaches, I have tried and experimented with a few content management systems(CMS) in order to find their strengths and weaknesses. These CMS being Joomla, Drupal, SilverStripe and Wordpress. Then continued to developed a set of prototypes in which the research findings were applied to aid in the usability of both the front-end admin area and the back-end website. 


\section{Acknowledgments}

This thesis wouldn't be the same without the people around me.

I would first like to thank my thesis supervisor Walter Langelaar, Programme Director in Media Design at the School of Design, Victoria University of Wellington. Mr Langelaar consistently allowed me to work on this thesis and creative output independently, while at the same time steering me in the right the direction with much needed critical feedback, support and idea development.

I would also like to acknowledge Laila Faisal from Student Learning, Victoria University of Wellington. Laila helped me to develop the overall quality of my literature as well as providing intellectual and moral support week after week, and for that I am grateful.

Furthermore, I would like to thank Ryan Achten for his help and contribution in the UI design of the website. My friends Andrew Hillstead and Pablo Gonzales and cousin Joey Sakamoto for all their moral support.

Finally I would like to express my very profound gratitude to my mother Amalfi Trujillo for providing me with unfailing support and continuous encouragement throughout my years of study and through the process of developing this thesis and creative output. Thank you.

Juan Sebastian Paz 


\section{CONTENTS}

$\begin{array}{lll}1.1 & \text { Introduction } & 7\end{array}$

$\begin{array}{lll}2.1 & \text { Literature review - online collaboration } & 9\end{array}$

$\begin{array}{ll}2.2 & \text { Literature review - usability design } \\ \end{array}$

2.3 Literature review - human centred design 15

$\begin{array}{lll}2.4 & \text { Research methods } & 16\end{array}$

3.1 Introduction to the content management system 18

$\begin{array}{lll}3.2 & \text { Joomla CMS } & 19\end{array}$

$\begin{array}{lll}3.3 & \text { Drupal CMS } & 20\end{array}$

$\begin{array}{lll}3.4 & \text { SilverStripe CMS } & 21\end{array}$

3.5 Wordpress CMS 23

4.1 The Project - front-end 25

$\begin{array}{lll}4.2 & \text { The prototypes } & 30\end{array}$

$\begin{array}{lll}4.3 & \text { Analysis } & 33\end{array}$

$\begin{array}{lll}5.1 & \text { Reflection } & 38\end{array}$

$\begin{array}{lll}5.2 & \text { Output } & 40\end{array}$

$\begin{array}{lll}5.3 & \text { Conclusion } & 41\end{array}$

5.4 References 42 


\section{Introduction}

This thesis focuses on creating a user-friendly experience for a sustainable and collaborative academic community website which showcases different work. The "users" in this case covers front-end users which include; prospective students, current students and their friends and family, as well as; administrative users student representatives, lecturers, tutors and course admins.

Prior to my thesis, I had an idea of making a self-sustainable School of Design website which was run by students and made by students. The aim of it was to generate a collaborative community where students will have their work stored and curated. By the end of the students degree they will have a safe location to access their stored work. Because of this idea, I joined a group of graduates who had a similar goal.

As a result, one of the contributors lecturer Walter Langelaar created a group in a VUW internet design course. During this course, I was a tutor. We included a project for students to continue on the development of the website under our supervision. This leads the students to learn, design and develop while collaborating with each other. This collaboration was done through the use of external software such as GitHub and google docs. After the end of the paper, I saw the advantages of what a group of students can design and develop as a community for a website that they will feel connected and showcase their own work in the future.

The research in this thesis focuses on two different categories: Online collaboration and usability design. The research into online collaboration explores a few themes. These themes include the culture, guidelines, lessons and the social aspects of online collaboration. As for the research into usability design, this research focuses on 
applying the knowledge acquired during the online collaboration by looking into the themes of the psychology of design, user experience for the web and how to design for forms.

After the research was done I understood some of the challenges and downsides of both the usability of most admin areas and the usability challenges that front-end designs have for displaying data. Some of the downsides for the admin area shows in their lack of instructions, the lack of available types of fields and lack of documentation. As for the front-end design challenges they mainly show in the availability of content. How to get to the user's destination with the least amount of clicks.

But to properly understand most CMSs (Content Management Systems) This thesis experiments with some of the most popular CMSs in the market, and shows in more detail their strengths and weaknesses of their out of the box state. These content management systems being: Joomla, Drupal, SilverStripe and Wordpress. By understanding these challenges and the testing and exploration of some content management systems and throughout an iterative approach at designing new interfaces and the development of prototypes to attempt at solving these challenges for an academic websites run by the online collaboration of staff and students. During this process, some solutions to such challenges were found an applied.

How to make user-friendly experiences that leverage online collaboration? This is the topic of this thesis. I have spent 12 months trying to answer that question while designing and developing an experimental custom built WordPress content management system and a new interface for the web user to be able to get the information needed within very few clicks. 


\section{Literature review}

This research focuses on the approaches on usability within web design and development. Understanding the relationship between the user and the machine, how the web is used and the importance to put human needs, capabilities and behaviour first. However, to apply this knowledge to an academic community project like the School of Design website, research into online collaboration was done to understand what online collaboration is, the social aspects of it, the culture around it and the lessons that have been learned.

\section{Online Collaboration}

Online collaboration is a term used to describe a group effort of creating content online. A good example of an online collaboration is Wikipedia, where millions of people from around the world have created the largest free online Encyclopedia.

This research focuses on the themes of social aspects of online collaboration, Online collaboration guidelines, online collaboration as culture and the lessons from online collaboration. The research on these themes will help to find the strengths and weaknesses that online collaboration brings to a development environment lead by an academic community. This research will demonstrate how to address the collaboration and attribution of the people who take part in it.

In order to design and develop a system that focuses on online collaboration, the social aspects of this type of collaboration must be researched. To understand how the aspects of online social collaboration work, the online book Collaborative Futures was selected for this theme as the book itself was created by many different authors from different parts of the world and done in a sprint of six months all by collaborating and 
generating the content. The authors state that no creative act is strictly individual as it relies on sources and style of those which preceded it. (collaborative future), Legal methods have been required to keep this collectivity in control but as we are in a new era of digital production these methods will have to change, as it will no longer be enough to keep up. Collaborative future talks about treating open collaborations like any normal romantic relationships, as open collaborations are based on mutual trust, and trust alone can be too fragile social fabric to support human interaction. With this in mind, we can explore how terms such as sharing, openness, user-generated content and participation have become so mainstream that they tend to be misused.

Collaborative futures also talks about user-generated content and social media creating a tendency for confusion between sharing and collaborations. An example of this is blogging and media sharing as it does not directly lead to collaboration, as the author is directly attributed with it and the work is singular even if it's shared and has a free license on it. These are examples of what is not considered as collaborative work, but there are examples of collaborative work such as edits in wiki pages or commits to a version control system such as Github. As mentioned previously we used Github and Google Docs to facilitate this collaboration between the students to achieve their objective. This objective being the task to create a few template pages for the school of design website using a SilverStripe framework as a content management system.

With the previous theme, helping understand how online collaboration should be treated in social terms. The online collaboration as culture theme helps to understand the relationship of the human and the computer and the culture around the community. For the reading Behind the Blip : Software as culture helps us understand that Human-Computer Interaction $(\mathrm{HCl})$ is one area that should be turned to, and as Fuller (2003) states "this is, after all, the point at which the machinations of the computer are compelled to make themselves available in one way or another to the user"(p. 12). The way the computer makes able such use and assumptions about what possible interactions are available are both cultural. Fuller (2003) also showed that $\mathrm{HCl}$ works 
and it is productive because it belongs to a long line of disciplinary idealisations of the human that nevertheless has the capacity to latch onto flesh. He gives an example that when it comes to the most suitable combination of ergonomics and information-design to ensure that a pilot can drop bombs or stockbrokers can move funds in an efficient, information-rich, graphically and emotionally uncluttered manner, $\mathrm{HCl}$ delivers the goods. The flaw in this is that there is a limit to human model but in Alan Cooper's Approach to interface design, works by establishing a number of stereotypical users of a system that is later imagined as full system users to help design an interface.

Establishing the importance of the culture and human-computer interaction, we can move into the theme of Online collaboration guidelines. During these last few years with the rapid advancements of web technologies, many new types of software tools have been developed to facilitate online collaboration as it no longers requires designers and developers to be physically next to each other and be able to complete tasks from a distance. This new type of work has been adopted by many communities to develop free and open source projects (F/OSS). With this in mind the journal Collective Development in Open source communities: an active theoretical perspective on successful online collaboration show that online collaboration is organized without strong predetermined rules or central authority, which is why coordination and ways of organizing cooperation become crucial elements of collaboration. The journal also argues that online cooperation is not just about task coordination but a question on how to overcome tension that comes from the alignment of strategic activity and individual action in a dispersed group. It also talks about the term Coat Tailing which is used to denote the parallel pursuit of individual and collective objectives. Hemetsberger and Reinhardt (2009) states "Coat tailing means to inextricably bind together individual action and collective activity through careful design of complexes of technological, mental and cultural artefacts"(p. 987). Yrjö Engeström(2004) says that "collaboration among people with such a varying expertise necessitates a dynamic, 
diabolic relationship between multiple actors; it is a relationship characterized by collaborative and discursive constructions of the task."

With this in mind and recognising that there will be a different range of skills in students, I aim to integrate and develop guidelines for the objectives of creating a new iteration of the website. With clear goals, the collaboration between the community will flow with no complications. Having clear objectives will solve the problem of conflicting goals between different actors these conflicts can impede the pursuit of the activity and achievement of the task. As it is easy to lose sight of the overall objective in large collaborative online projects.

With this research I have looked at the social impact of online collaboration, Online collaboration as culture, guidelines for collaboration and now to continue with the last theme; lessons from online collaboration. As mentioned above the fast development of web technologies have created many groups and communities that already tackle online collaboration. Because of this, there is an increase of interest in the study of knowledge flows in online communities. The authors Zablith, Faraj, and Azad (2016) highlight in the journal article Organizational knowledge generation: lessons from online communities that the boundaries between internal and external organizational knowledge are disappearing mainly due to the extensive use of online-based platforms to support organizational operations. They also believe that this will affect the activities of knowledge management in today's businesses. As McDermott (1999) states, "organizations are becoming enmeshed in electronic networks" resulting in the organization's knowledge gradually becoming part of the online conversation. Meaning that the knowledge as a source of competitive advantage of the organization that is to be secured as a valuable resource is now becoming a model where knowledge is now something that is free-flowing. It forms a community of enthusiast is a radical change that is affecting most firms. With these new changes, I agree and disagree with these new changes. I agree that the information should be free-flowing but at the same time 
disagree that some of the knowledge should be kept as a source of competitive advantage. In the development of the thesis, I think while all of the development should be done in collaboration by students it should be curated by members of the staff as in the end it is a website that belongs to the school of design in Victoria University.

These four themes have explored some of the most important areas for the research, as social aspects of online collaboration help understand the meaning and barebones guidelines on how to tackle online collaboration, this being the understanding of the relationship that will generate the collaboration of students within the development of a new iteration of the website. With this understanding, we can now explore the cultural impact on online collaboration and concentrate on the proper guidelines and objectives that the community need to have in order to be an efficient collaboration between this group. These goals and objectives will need to be done by members of staff so they can properly curate the content and aims of each iteration as seen by the lessons of online collaboration. 


\section{Usability Design}

So far this research has focused on understanding online collaboration, its guidelines, the lessons and the culture that it has created on the internet. This knowledge helps us understand how to properly set up a system that uses online collaboration as a focus. But to be able to properly deliver this focus, research into user experience design needs to happen to develop a content management system that facilitates the online collaboration of an educational website. This research needs to focus on the following themes: 'The psychology of design' to understand the interaction and relationship between the user and the product, 'user experience for the web' as this product is an online application, and 'designing for forms'.

Two of the most important characteristics of good design are discoverability and understanding discoverability (Norman, 2013). This refers to the relationship of the user and the product as well as the behaviour of how the user will interact with the product. The user will need to be able to know what actions are possible and how to perform them. They will need to know how the product is supposed to be used and how to control it.

Due to the recent rapid advancements in technology, new experimental ways of interaction have risen. Specifically, the advancements in computer electronic devices. During these advancements of technology, the field of $\mathrm{HCl}$ or 'human-computer interaction' was created. 


\section{$\mathrm{HCl}$}

Human-Computer Interaction $(\mathrm{HCl})$ is a multidisciplinary field of study focusing on the design of computer technology and, in particular, the interaction between humans "the users" and computers. (Carroll, Zahirovic, Lowgreen, Hassenzahl, Erickson).

\section{The Multidisciplinary Field of $\mathrm{HCl}$}

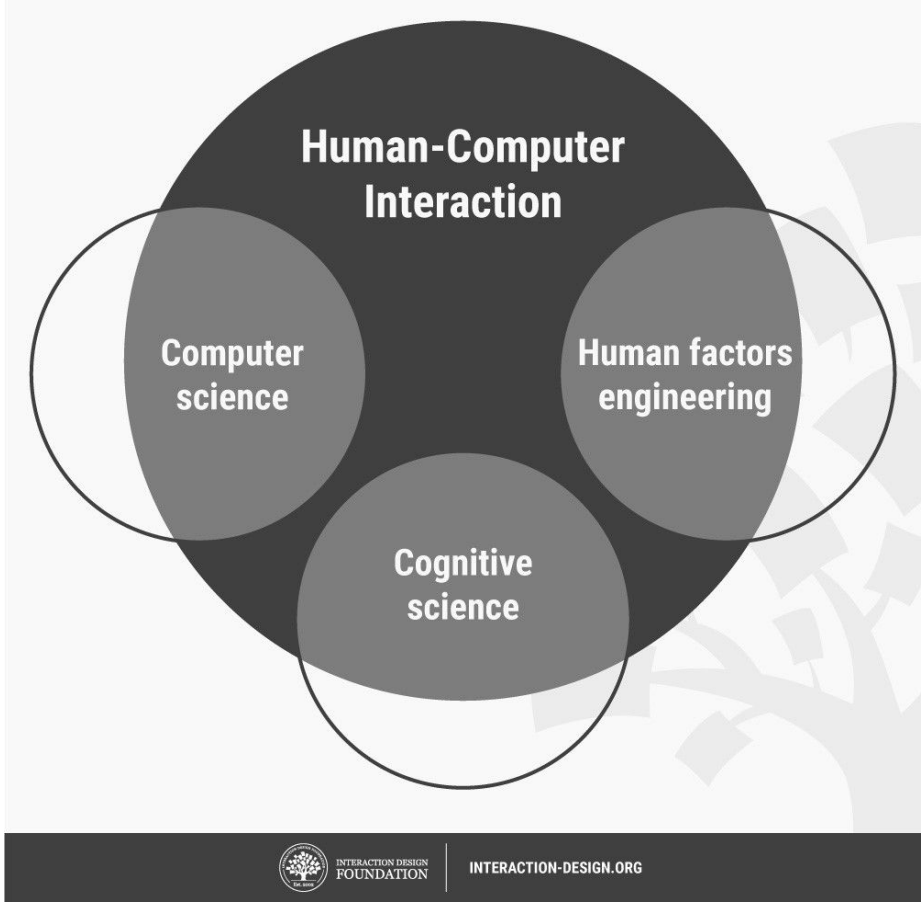

Figure 1. Human Computer Interaction. Retrieved from The Encyclopedia of Human Computer Interaction, by Carroll, Zahirovic, Lowgreen, Hassenzahl, Erickson, from https://www.interaction-design.org/literature/book/the-encyclopedia-of-human-computer-inter action-2nd-ed/human-computer-interaction-brief-intro

$\mathrm{HCl}$ emerged in the 1980s with the increasing demand of personal computing, just as machines such as the Apple Macintosh, IBM PC 5150 and Commodore 64 rapidly began turning up in homes and offices all over the world. 
For experts in specialized tech environments, the need to create a human-computer interaction that was also easy and efficient for less experienced users became increasingly vital. (Carroll, Zahirovic, Lowgreen, Hassenzahl, Erickson).

$\mathrm{HCl}$ original academic place was computer science, and its original focus was on personal productivity applications such as text editing and spreadsheets. Since then, the field has been growing, diversifying and expanding to encompass visualization, information systems, collaborative systems, system development process and other areas of design.

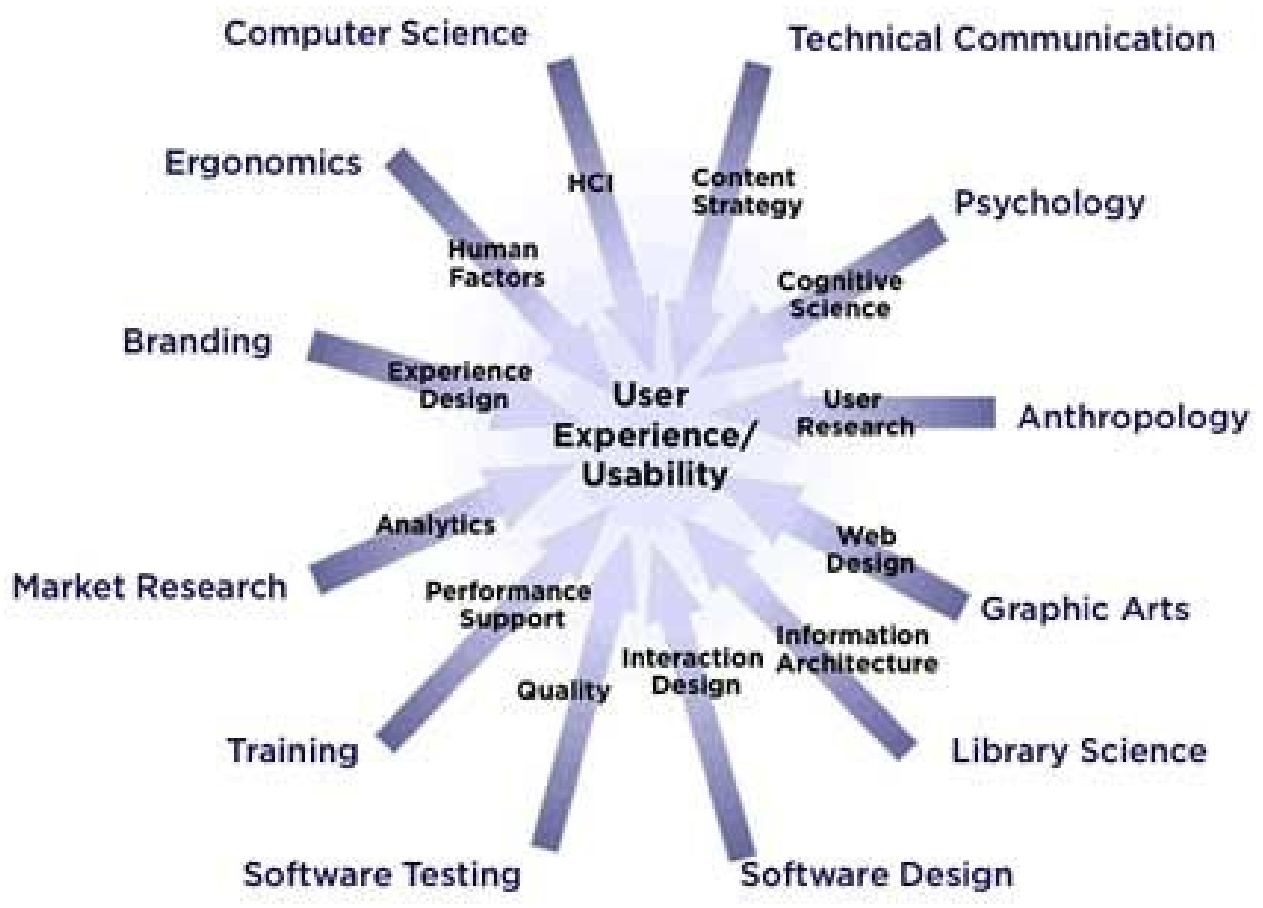

Figure 2. User Experience/Usability. Retrieved from The Encyclopedia of Human Computer Interaction, by Carroll, Zahirovic, Lowgreen, Hassenzahl, Erickson, from https://www.interaction-design.org/literature/book/the-encyclopedia-of-human-computer-inter action-2nd-ed/human-computer-interaction-brief-intro 
One of the major applications of $\mathrm{HCl}$ in the early days was in the development of the 'desk metaphor' (defined as a desktop graphical user interface) popularized by Apple Macintosh: where the user no longer needed to type text-based commands to do certain tasks. Before this development, all interactions between the user and the computer was done via typing in the command line. It was now able to interact by clicking, double-clicking and dragging files and folders around. As this was a new technology and a very experimental way of interaction, it came with a lot of user problems. the product was not as easy to learn as it was advertised. Although as this new way of interaction was introduced to the public it captured the imagination of the designer and gave way to new improvements and possibilities.

While the human-computer interaction approach has great results when done right, it also comes with its deficiencies. As Norman(2013) states "It is not our duty to understand the arbitrary, meaningless dictates of machines." (Norman, 2013).

These deficiencies being that most of the design is done by engineers who are experts in technology but limited when it comes to understanding people. Engineers are trained to think logically. As a result, they generally assume that all people think this way. Therefore, they designed their machines or systems accordingly.

Since the emergence of human-computer interaction, a few decades have passed. During these decades many new developments in technology have emerged which have outpaced the advancements in design. New applications and methods of interaction continue to arise and evolve. The book 'Design of Everyday Things' states that people are frustrated with everyday things, from increasing complexity of automobile dashboards to all systems of entertainment and communication. It also states that design has got better as there are many books and courses on the topic. 
But even with all these improvements, the changes in technology outpaces those of design.

\section{Human Centred Design}

The solution to the frustration of human-computer interaction is Human-Centred Design (HCD). Human Centered Design is an approach that puts human needs, capabilities, and behaviour first and then designs to accommodate those needs, capabilities, and ways of behaving. (Norman, 2013). Norman(2013) also states that Good design starts with an understanding of psychology and technology. Good design requires good communication, especially from machine to person, indicating what actions are possible, what is happening, and what is about to happen. Human-centred design is a design philosophy. It means starting with a good understanding of people and the needs that the design is intended to meet. (Norman, 2013).

With some knowledge into design approaches, I can begin to apply them in a web setting. One of the major advancements in technology has been the invention of the world wide web and the increasing developments of web technologies and web browsers. These advancements have given rise to many new challenges when it comes to design for the web.

"DONT MAKE ME THINK" - first law of usability

For instance, it means that as far as is humanly possible, when I look at a Web page it should be self-evident, obvious and self-explanatory. The web these days is often used for quick searches and experiences, the user wants to obtain the information as quickly and efficiently as possible with minimal effort required. The user also wants to get to their destination with as few clicks as possible, so having good content distribution and proper navigation is essential for good web design. (Krug, 2014) 
Another fact to keep in mind is that web users don't 'read pages'. We scan them. Why do we scan them? According to Krug(2014), we do that because we are usually on a mission as most web use is to try to get something done quickly, we know we don't need to read everything. As we are only interested in a fraction of what is on the page, and we also scan web pages because we are good at it, as its a basic skill we already have, when we learn to ready we also learn to scan. We are used to scanning newspapers, magazines and websites such as Reddit, Tumblr, and other social media applications.

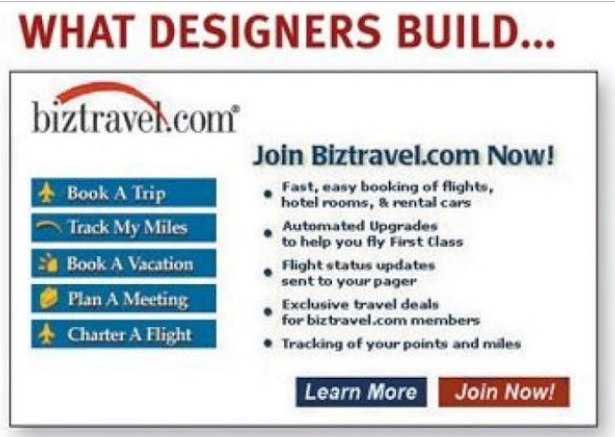

\section{WHAT USERS SEE...}
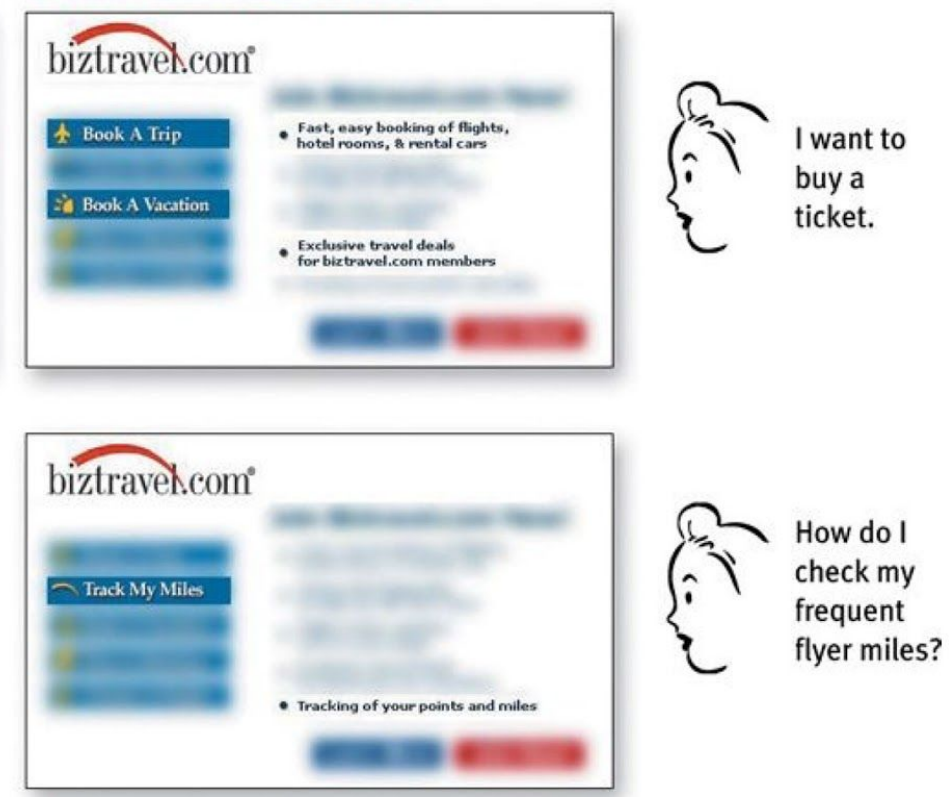

Figure 3. How we scan web pages. Reprinted from Dont make me think (p. 34), by Steve Krug.

We get it! Users scan, while designing web pages we tend to assume that users will come to the website and scan the page, consider all options and choose the best one. In reality, we don't choose the best option. According to Krug(2014), we choose the first reasonable option, this is known as satisficing. As soon as we find a link that seems like it might lead us to the information we want. We will most likely click it. Why 
do we do this? We are usually in a hurry and there is not much of a penalty for guessing wrong as the back button is only a click away. (back button is the most used button in web browsers)

One thing is clear, we must design for scanning in mind. $\operatorname{Krug}(2014)$ states that the following are some of the things you must do to make sure your users see and understand what they need to do.

- Take advantage of conventions

- Create effective visual hierarchies

- Break pages up into clearly defined areas

- Make it obvious what's clickable

- Eliminate distractions

- Format content to support scanning 


\section{Research methods}

Originally, When I started my thesis, I was planning my output would be to design and develop a completely new CMS that had online collaboration and social coding in mind. For this, I would have to develop not just a CMS but also modules for an online repository, text editor, quality control and task coordination. But to completely create a system like this from scratch it will be out of scope for my thesis, It will most likely take a lot of time, a team of people and a good amount of money. So I decided to rather than building a system from scratch I will instead focus on researching and making both a good frontend in which users will find the content in a new and fun navigation and an admin area in which all users will be able to create all the content for it in an easy to use way.

My output is going to have two separate portions, the frontend design in which all data from student work and new types of playful navigations is going to be displayed and an admin area in which the users will enter the data for the whole website. For the design process, I will be using the approach of HCD methodology. This approach puts human needs, capabilities and behaviour first. This methodology will fit the ethos of the thesis since I aim to make the admin area as user-friendly as possible and to make the front-end website a playful and interactive experience for all users. as Norman (2014) states, Good design starts with an understanding of psychology and technology.

But not everything in my thesis is design, I am also developing a custom build CMS. for this development I need a technological approach to help get to the desired output. As there are multiple content management systems in which I can choose and develop from. I need to make a few prototypes to decide which of the content management systems to choose from. For this approach, I decided to research into more experimental methods as most of what I am doing is of experimental nature. From the 
book inventive methods I found an interesting method to software development that has an iterative approach to it. This method being Probes, probes are a method for developing richly textured but a fragmented understanding of a setting or situation, their purpose is not to capture what it is so much as to inspire what might be. (Lury \& Wakeford, 2015) 


\section{Introduction to the content management system}

From the start of this project, one of the most important features to focus on was the ease of use for the admin area. As this admin area is going to be used by both the staff and selected students. Research into usability design had to take place. The importance of the usability not only had to be for the end website itself but mainly in the admin area. For this, a CMS had to be installed and developed upon it. A CMS or Content Management system is quite normal these days. As they are widely used across many web projects. Because of this popularity, there is a large number of options to choose from. But the main points for selecting such CMS is the ease of use, the freedom for a developer to change many of the functionality of it and last but not least, the social aspect of it, or the amount of user-generated plugins that expand the use of such CMS.

\section{Market share}

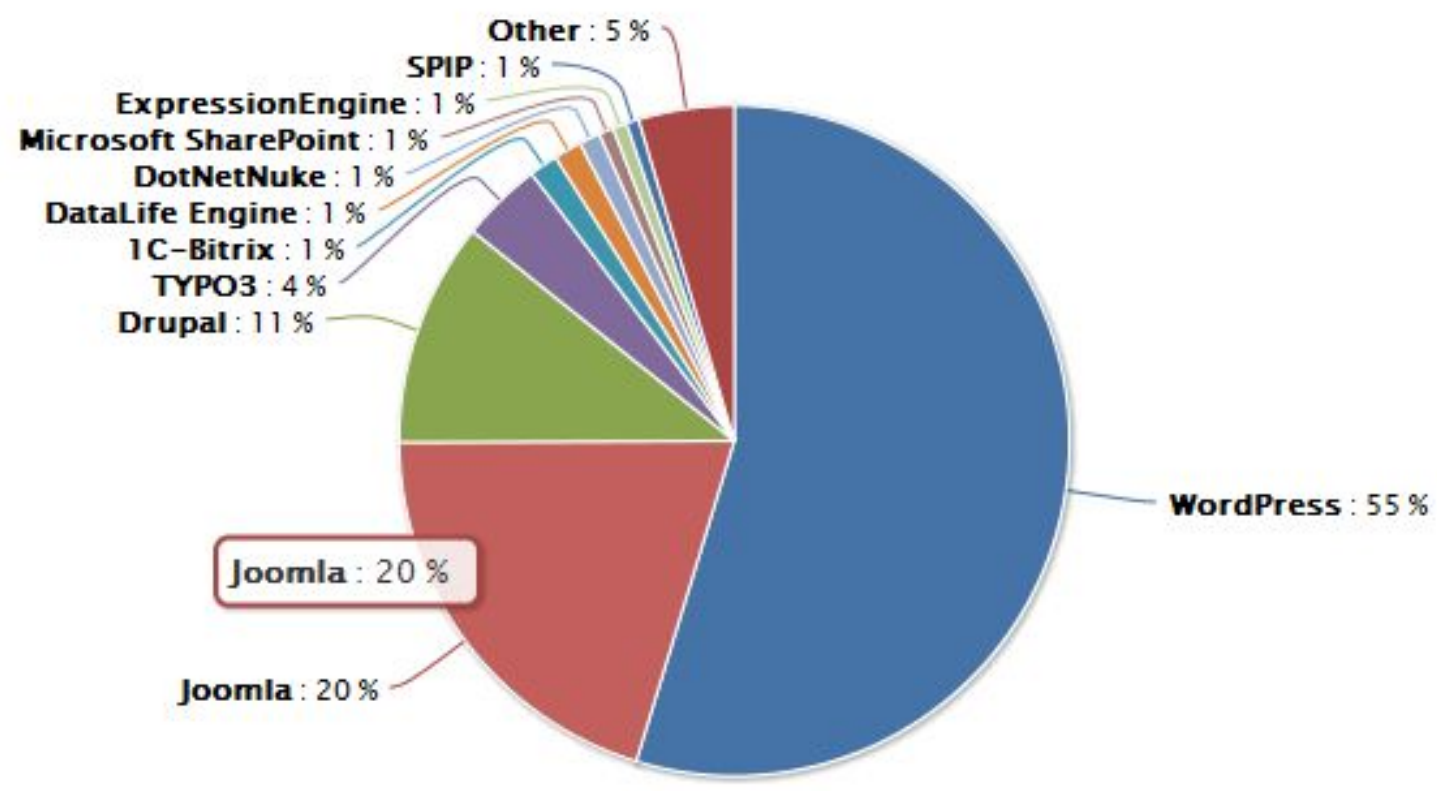


Figure 4. CMS web usage statistic. by GAVICKPRO TEAM Retrieved from https://www.gavick.com/blog/wappalyzer-and-cms-including-joomla

From the many options of content management systems to try only a few were selected. These few were Wordpress, Silverstripe, Joomla and Drupal. For all of these selected systems an instance each was installed and reviewed for its strengths and faults. These strengths being the ease of use, the freedom for development, the social aspect of it and the amount of user-generated content and documentation for it.

Before we get into what makes each CMS unique and why some were chosen to iterate upon and somewhere not, we'll cover some of the things they have in common. We should point out, however, as time has gone on they have become increasingly similar, and they will likely continue to do so.

Like every other CMS, each of these has both a front and back-end: the website shown to visitors, in this case, it is the school of design website where users can view announcements and student work, and the other an administrative console and an easy to edit presentation layer. Unlike every other CMS, each of these is an open-source content management system. This means they are free to use by the community.

They are all built for use on a LAMP stack (server using Linux, Apache, MySQL and PHP), which is the most common web server setup. However, they can be forced to use others (Drupal is more readily able to use other databases) and are built to be capable, flexible, and most importantly, freeing and empowering. 
Joomla

Joomla is one of the web's most popular open source CMS and one of the most used open source CMS worldwide. Joomla is the second most used CMS in the entire internet. But not widely used with high traffic sites.

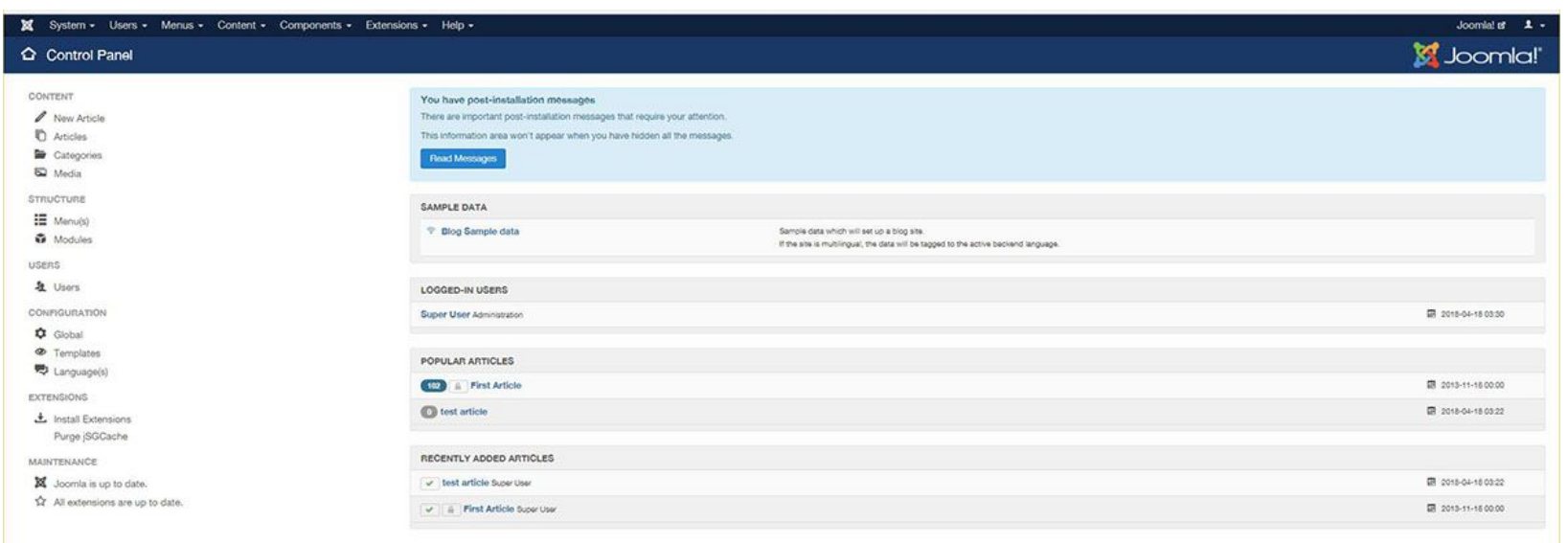

Figure 5. Joomla dashboard CMS. Screenshot taken from local machine.

Getting started with Joomla can be a bit overwhelming as straight from the box there is a large number of options that are available for the user and two separate menus one horizontal and a vertical. Both of which contain access to both different and same functionality. But that adds to the confusion. When looking at the webpage it should be self-evident. Obvious. Self-explanatory (Krug, 2014) even with a complex system such as a CMS. But some aspect of it are laid out in a way that the user can see the option to create a new article as a first thing it sees. Another blot on Joomla is the plugin and extension repository. Although there are more than 7700 extensions available, most of them are restricted in their functionality and many are outdated. The lack of regularly updated Joomla extensions has hit the overall functionality of the CMS hard. Because of this negative factor, Joomla was not selected as a software to develop a prototype in. 


\section{Drupal}

Statistics say Drupal is the third most popular $\mathrm{cms}$ in the web. But while that only indicates about $5 \%$ of the web. Statistics also say that Drupal has a big percentage on its use on high traffic websites. Drupal targets its platform to developers, marketers and agencies, for developers it leverages an API-first architecture, robust configuration management and extensibility to build on the web.

\begin{tabular}{l|l|l|l|l|l|l|l} 
Home Administration & & \\
Dashboard & DASHBOARD & CONTENT & COMMENTS & CATEGORIES & USER ACCOUNTS \\
\hline
\end{tabular}

Take Total Control.

Welcome to your administrative dashboard. Edit this panel to add more content panes here, or configure those provided by default. Views content panes can be created for you automatically according to your Total Control Settings. Make this dashboard the perfect hub for all your administrative needs.

\section{Create content}

- Add new Article

- Add new project

- Add new Video

\section{Content overview}

- 58 Article items with 28 comments

- 62 Basic page items

- 0 project items

- 0 Video items

\section{New User Accounts}

- testuser 111 | Created: $11 / 30$ | edit

- testuser111 | Created: $11 / 30$ | edit

- testuser111|Created: $11 / 30$ | edit

- testuser111 | Created: $11 / 30$ | edit

- testuser111|Created: $11 / 30$ | edit

\section{New Content}

- Augue Consequat Euismod Jus Article by: test on: 11/30 1:31pm

- Aptent Paratus

Basic page by: Anonymous (not verified) on: 11/30 9:43am

- Amet Iriure Mos

Basic page by: root on: 11/30 6:07am

- Conventio Os Tation

Article by: root on: 11/30 6:06am

\section{Administer Menus}

\begin{tabular}{|c|c|c|c|}
\hline MENU & OPERATION & & \\
\hline Main menu & Configure & Manage links & Add new link \\
\hline Navigation & Configure & Manage links & Add new link \\
\hline
\end{tabular}

Menu administration

Administer Content types

\begin{tabular}{|l|lll|}
\hline CONTENT TYPE & OPERATIONS & & \\
\hline Article & Configure & Manage fields & Manage display \\
Basic page & Configure & Manage fields & Manage display \\
project & Configure & Manage fields & Manage display \\
Video & Configure & Manage fields & Manage display \\
\hline
\end{tabular}

Content type administration

Administer Taxonomy

\begin{tabular}{|c|c|c|c|}
\hline VOCABULARY & OPERATION & & \\
\hline $\begin{array}{l}\text { Categories: } 3 \\
\text { categories }\end{array}$ & Configure & $\begin{array}{l}\text { Manage } \\
\text { categories }\end{array}$ & $\begin{array}{l}\text { Add new } \\
\text { category }\end{array}$ \\
\hline
\end{tabular}

Taxonomy administration

Figure 6. Drupal dashboard CMS

Drupal was initially released in January of 2001, but it was originally conceived to serve as the framework for a small site that housed information for some students at the 
University of Antwerp. It evolved quickly following its release as an open source framework. The platform itself is designed to be incredibly flexible to serve not only as a platform for a website, but also for forums, blogs, network sites, and other applications. The system is a little more technically agnostic than the other systems in discussion, as it can work with a myriad of databases.

As mentioned before one of the main targets of this CMS is the developers. So most of the design features of vanilla Drupal come almost as a blank slate to be customized on all levels. Therefore it's out of the box interface is clunky with not much usability focus. So if a user visits the admin area of this $\mathrm{cms}$, this user will have a hard time trying to get started. While Drupal lacks in some areas, it is very strong in its development freedom, it is very customizable and a user can create a good custom admin experience.

The user roles in Drupal differ greatly from those of Joomla and WordPress, as Drupal user roles are entirely customizable. You may enable and disable functions and access to content areas based on user roles. For that reason, Drupal is one of the easiest systems to set up if you have a team with users who should only have permission to create or alter certain types of content.

These default settings have given Drupal a reputation for being difficult to use, but the reputation isn't entirely fair - the tool was built to act as a blank slate to be customized and turned into whatever you need it to be. These reputation has been unfair as Drupal by its default settings, as it was designed to be customized. However, these customizations bear a cost of both time and resources. For this reason, I consider it the most difficult of the three to use out of the box, 


\section{Silverstripe}

SilverStripe Ltd was founded in 2000 by three people; Sam Minnée, Tim Copeland and Sigurd Magnusson brought to life the SilverStripe CMS. Strongly believing that collaboration leads to better results, SilverStripe CMS was open sourced in 2006. This allowed developers and designers from around the world to shape and improve SilverStripe. Since this time a vibrant open source community has grown around the project and the company has expanded with offices across New Zealand, Australia and in the UK.

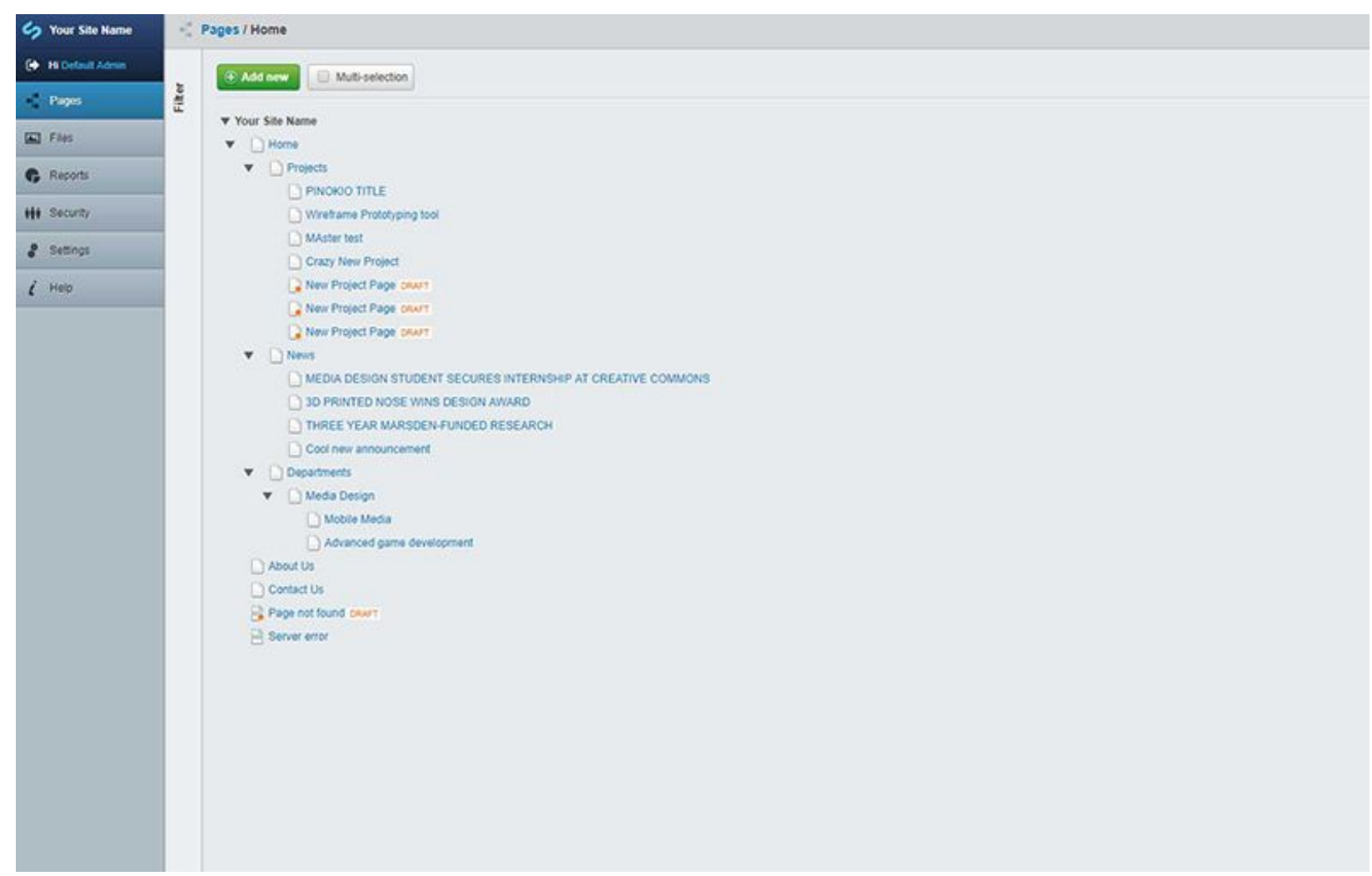

Figure 7. Prototype dashboard made in SilverStripe CMS. Screenshot taken from local machine.

While SilverStripe is not as popular worldwide in its use as the other systems in discussion, Silverstripe it is quite popular in the New Zealand web industry. 
SilverStripe consists of a framework and CMS, which can be used independently but probably you wouldn't them separately in most cases. It takes advantage of $\mathrm{OO}$ design, MVC, modern patterns, object-relational mapper etc. In terms of the community support, SS can't be really compared to the three giants we discussed above. The CMS is heavily AJAX-based, which is good for the most cases. Although plugin base (called add-ons or modules) are limited, it provides an amazing, lovely platform, logic and power for a developer.

The initial installation comes with a logical hierarchical versioned system called Site Tree, which provides a good launchpad. Grid-based data presentation on the admin panel looks very professional. The designed choices on the $\mathrm{UI}$ are very considered which helps the usability of the admin area.

Security is good. It has a nice permission/user management features coming out of the box. You can also extend its capabilities and features. Generally, SilverStripe is recommended for general purpose custom application development. SEO, social media integration, site search etc. are pretty decent - but you need some basic knowledge of configuration. None of them is a button to press in the admin panel. This takes us the some of the downs and ups of this system, The down being that is very developer heavy and the Positive being that is very developer heavy. With some coding experience, you can create a lot of functionality for the system, the CMS is very customizable but at the same time, the lack of documentation and poor plugin library brings a great system. For this reason, SilverStripe was chosen to develop a prototype with.

Security is good. It has a nice permission/user management features coming out of box. You can also extend its capabilities and features. Generally, SilverStripe is recommended for general purpose custom application development. SEO, social 
media integration, site search etc. are pretty decent - but you need some basic knowledge of configuration. None of them is a button to press in the admin panel. This takes us the some of the downs and ups of this system, The down being that is very developer heavy and the Positive being that is very developer heavy. With some coding experience you can create a lot of functionality for the system, the cms is very customizable but at the same time the lack of documentation and poor plugin library brings a great system. For this reason silverstripe was chosen to develop a prototype with.

\section{Wordpress.}

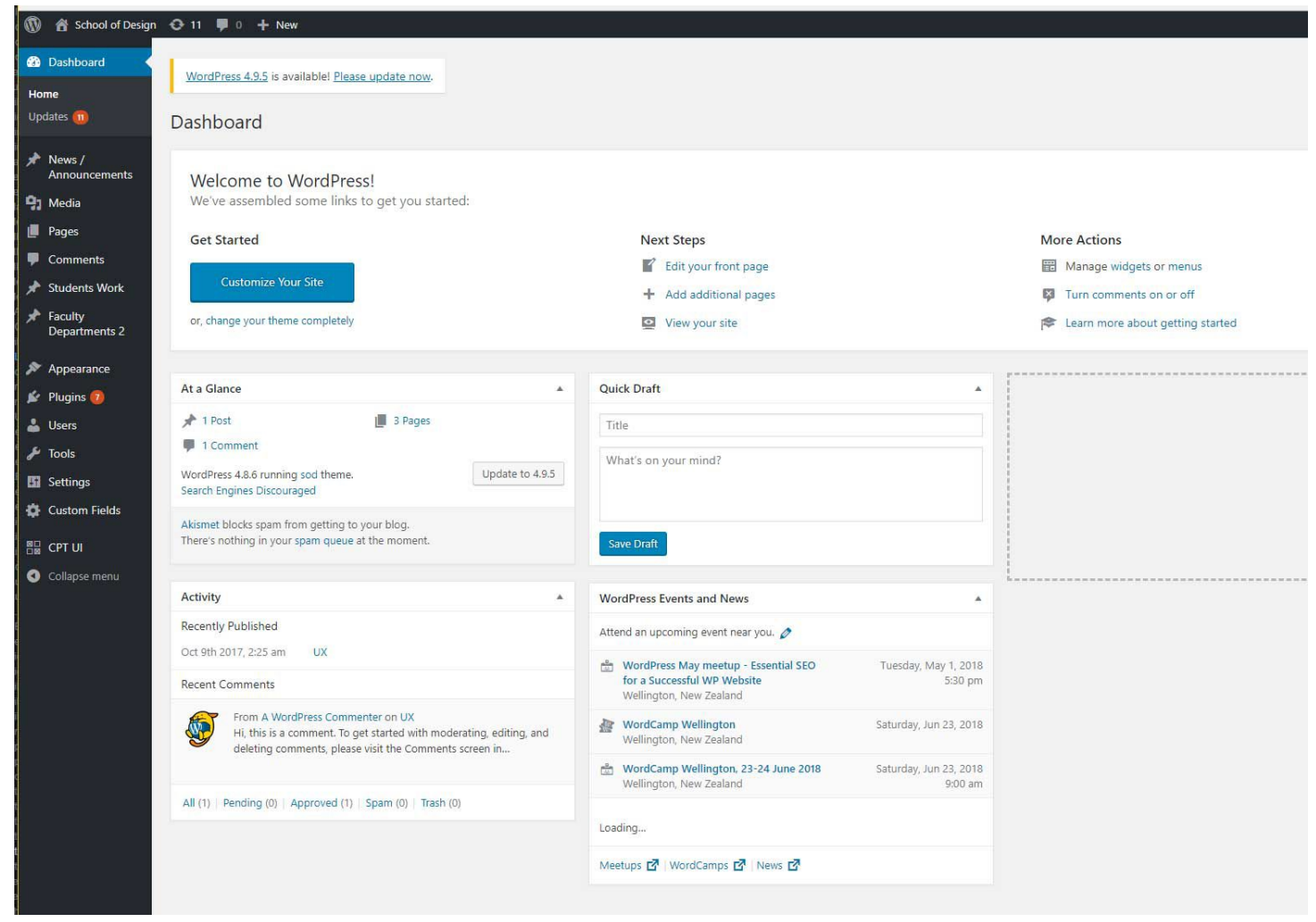

Figure 8. Prototype dashboard made in wordpress CMS. Screenshot taken from local machine.

WordPress was initially released in May of 2003 as an offshoot of b2/cafelog, which was originally a blogging platform designed to be added to existing sites. As 
WordPress grew in popularity, the developer community began to include more and more features commonly seen with more traditional content management systems.

WordPress is, as of the time, by far the most popular CMS on the web, powering about $59.2 \%$ of all of the websites that use a CMS, and about $28.3 \%$ of all websites.*

The core of WordPress' popularity lays within its ease of use. It started as a dedicated blogging platform meant it had to be intuitive and easy to use, as most of its users were lacking in technical knowledge, at least compared to a developer. For this reason, WordPress is incredibly intuitive, and users familiar with word processing tools like Microsoft Word will find WordPress simple to use from the start.

In its early years, this ease of use was a double-edged sword, because it made some compromises in terms of complex functionality. This caused some developers to deem it too lightweight to be a full-fledged CMS. However, in the past few years, WordPress has been more widely embraced by the developer community, in part because of its popularity and market share, but also because of some major technology improvements to the core platform itself.

While WordPress usability design is great, that's not its biggest strength. Wordpress biggest strength comes from the extensibility and its freedom for developer customization. And this goes hand to hand with a large amount of user-generated modules out there that improve upon the vanilla experience. This extensive community collaboration has made this system almost impossible to replace as it now runs $28 \%$ of the whole world wide web. 


\section{The Project}

To create a system that leverages online collaboration within an educational setting that displays student work and creates a self-sustainable community website. To achieve this I had to design and develop two parts, a design for a front-end website in which all the content can be accessed in just a few clicks. And a backend system in which all data is stored and curated in an easy to use admin system. Both of these systems combined makes the school of design website for Victoria University.

\section{The front-end}

Prior to my thesis, I had an idea of making a self-sustainable school of design website which was run by students and made by students. And the aim of it was to generate a collaborative community where students will have their work stored and curated and by the end of their degree they had a sort of portfolio ready. And because this idea I joined a group of graduates that had a similar goal. This group made a set of surveys regarding the importance of a school of design website. The target of this website was directed at staff, students, prospective students, potential research partners and other universities.

During this surveys, it showed that the students were more interested in having student work presented and information about what jobs are related to which courses. While the staff was more interested in a way to display the courses, an easy way to manage the resources for each course and also a way to keep and display successful projects. From the results of these surveys, there is one thing in common between the staff and the student, this being the importance to keep and display student work. Soon after the group disbanded. I decided to carry on with this idea and continued as my master's thesis. 


\section{First challenge}

From the start, I knew that one of the most important aspects of the project will be the ability to display student work and display course information. This was the first challenge for me to solve, a challenge of navigation. How will I effectively give most of the information on the whole website by only a few clicks, This was very important as stated by (Krug, 2014), users browse the web like they are on a mission. So the user wants to arrive at their destination very quickly. For example, this destination is a specific student work from an industrial design course. Most websites to achieve such destination will take plenty of page loads and user concentration. To solve this I had the idea to design the homepage with a filtering mechanic in which all content can be accessed by choosing categories without needing to load many pages.

To create a system that leverages online collaboration within an educational setting that displays student work and creates a self-sustainable community website. To achieve this I had to design and develop two parts, a design for a front-end website in which all the content can be accessed in just a few clicks. And a backend system in which all data is stored and curated in an easy to use admin system. Both of these systems combined makes the school of design website for Victoria University.

To create a system that leverages online collaboration within an educational setting that displays student work and creates self-sustainable community website. To achieve this I had to design and develop two parts, a design for a front-end website in which all the content can be accessed in just a few clicks. And a backend system in which all data is stored and curated in an easy to use admin system. Both of these systems combined makes the school of design website for Victoria University. 
Filtering system and tile mechanic

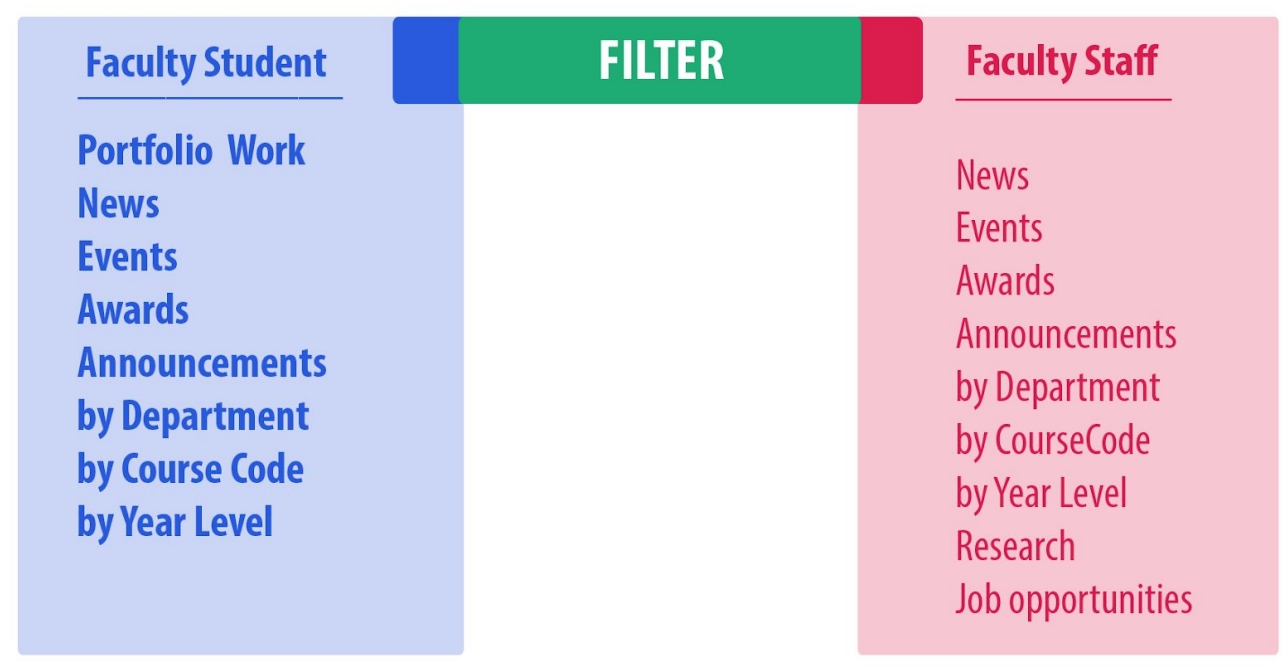

Figure 9. First iteration for the filtering menu. Screenshot taken from local machine.

This filtering system work by toggling two main buttons, one for the student and another one for the staff each containing a separate set of subcategories, once a category was selected then only the content related to it will be displayed in the homepage. I also designed the content to be displayed in tiles so it occupied a small space within the site containing only a tile, profile image and a brief information of the content. 


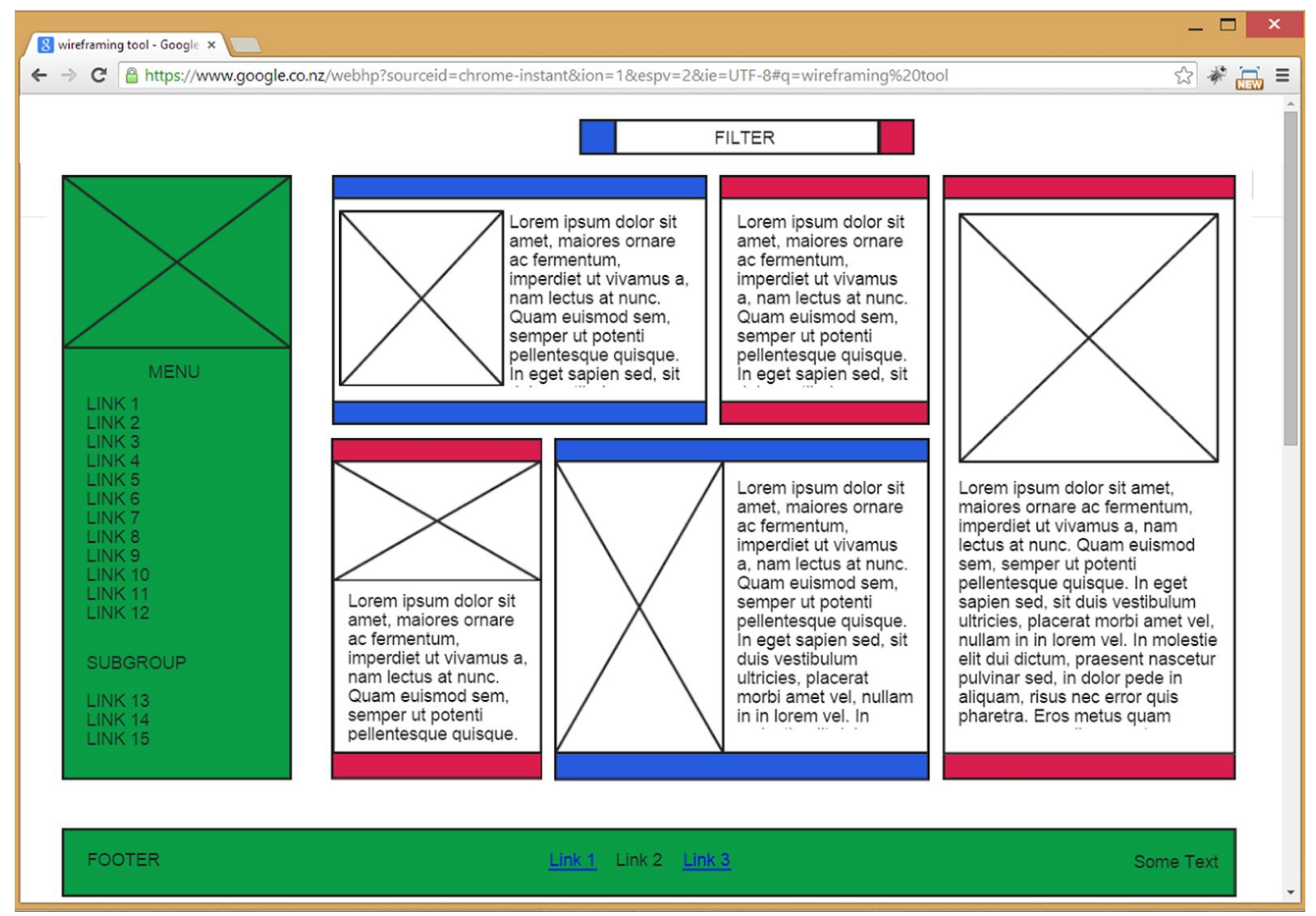

Figure 9. Wireframes for the first iteration of the homepage. Screenshot taken from local machine.

Originally during the first design iteration the tiles occupied too much space and didn't really contain much information for all the space it was taking and the only interaction was on the menu. So I went into designing a new iteration of this tiles to make it more interactive and take less space and contain more content. I designed it in a way so the tile can be interacted and when toggled the tile will "swap" sides to display extra information and a link to view it on full screen. However, the menu was still very complicated and didn't really solve anything regarding the ease of use and how to get to a destination in a few clicks. The next iteration had solved the problem and now all content can be displayed just by toggling six different buttons, these buttons being the different departments between the school of design.

- First year design

- Media design 
- Industrial design

- Culture and context design

- Master of design innovation

- Display all

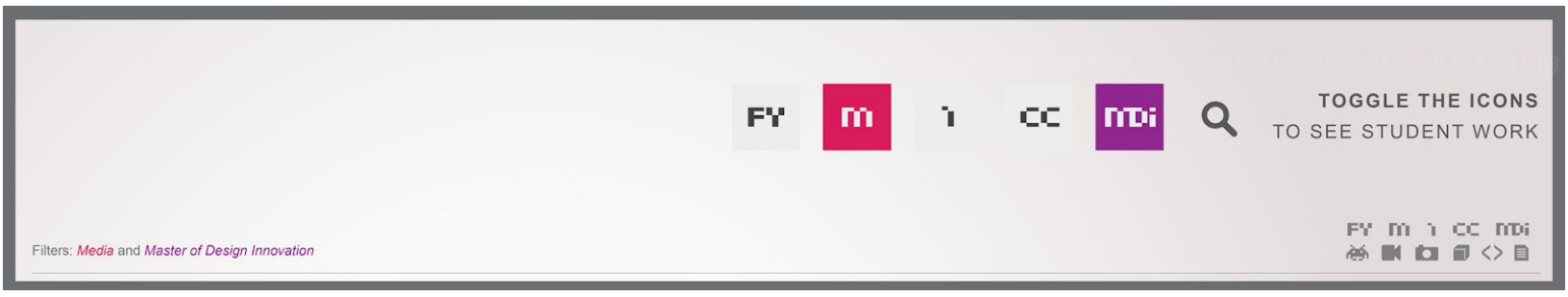

Figure 10. Final design for the filtering menu. Screenshot taken from local machine.

Not only could you only display content from media design but you could also mix and toggle multiple departments at once. The idea was that all content will be loaded on page load. And once a department was clicked only the tiles relating to that department will stay and the rest will disappear.

For the filter menu I took in consideration two main principles; Fitt's law that stipulates that the bigger the object and the closer it is to us, the easier it is to use(Gillian \& Holden, 1992). And the principle of visual hierarchy which refers to the arrangement or presentation of elements in a way that implies importance. With this combined, I made sure the most important elements of the whole website where the biggest. These being the content tiles and the filtering menu. 


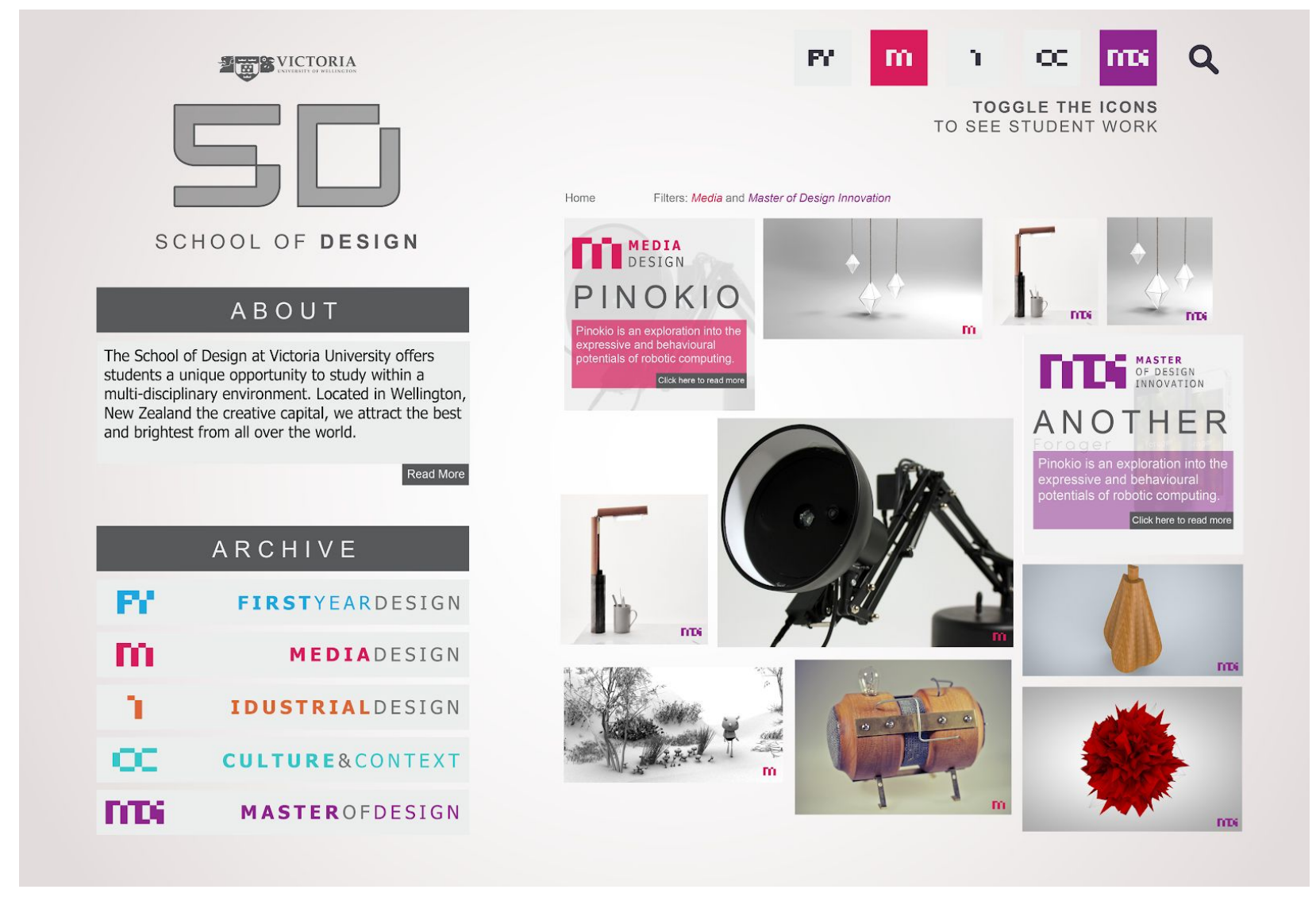

Figure 11. Iteration for the homepage. Screenshot taken from local machine. 


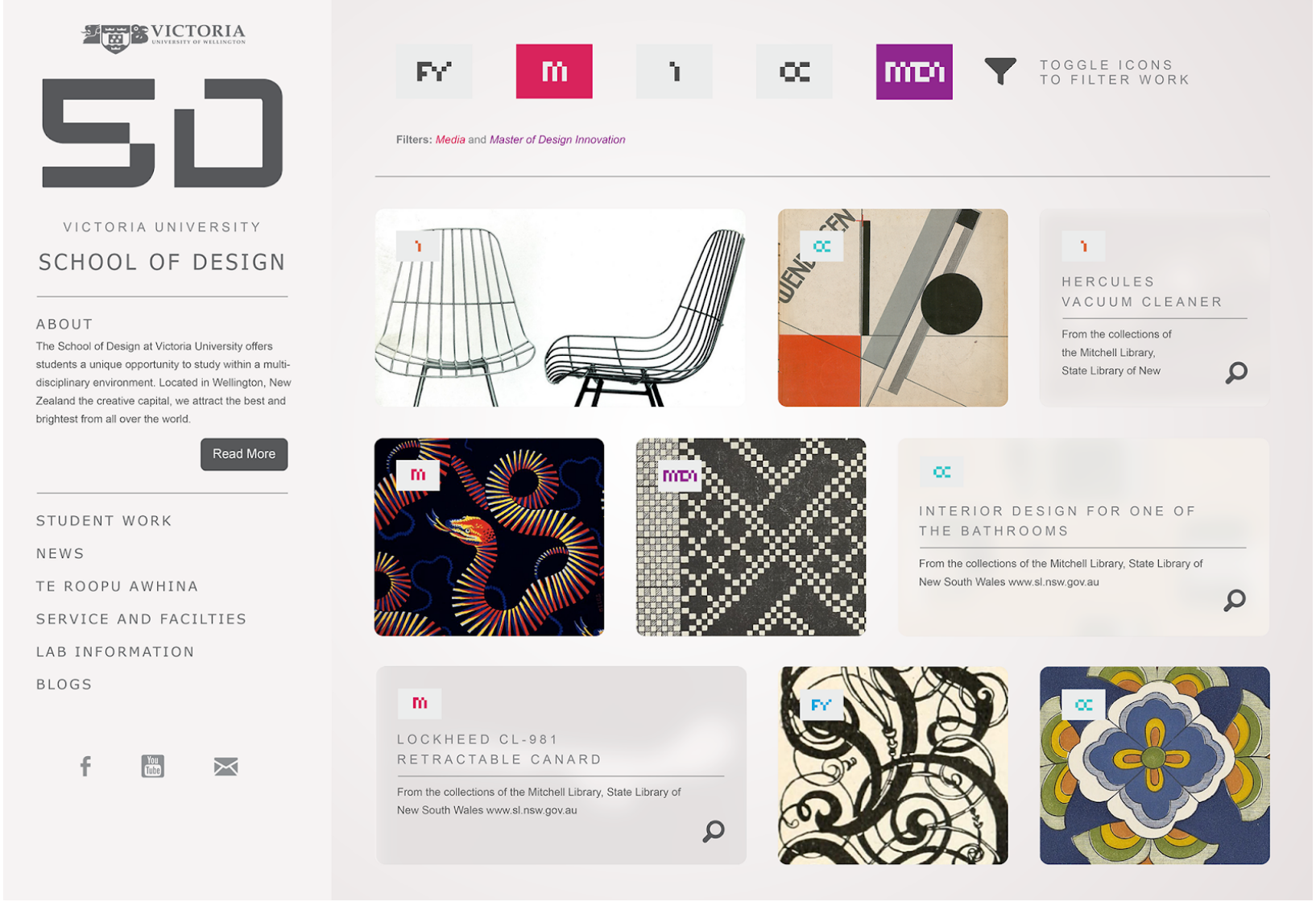

Figure 11. Final design for the homepage. Screenshot taken from local machine.

For the final design, the website is using the whole size of the browser both in height and width to be able to display the most content possible, even for those users who have $4 \mathrm{k}$ screens. The website itself It's also completely responsive, being able to adapt to all resolutions and devices.

So for those users who wanted to only see news and course information, another navigation was designed, this section taking place in the left side of the page. The rule of thirds was applied to the final design, the first third being the logo and secondary navigation and the rest for the filter menu and main content section. These main principles were applied to the design of the rest of the pages to keep the repetition and consistency of the design. 


\section{The Prototypes}

Having the design finished. Experimentations into the backend of the website had to begin. The initial experiments were on testing the most popular content management systems on the market, and choose a couple that out of the box had a user-friendly admin area, the ability to extend the capabilities of the software, the documentation for development and the better user-generated content available.

These tests were done on Drupal, Joomla, Silverstripe and Wordpress. And decided to choose Silverstripe and Wordpress to develop the prototypes to further experiment the ability to create custom work and better user experience for the admin area.

\section{Silverstripe prototype}

SilverStripe was chosen as a platform to develop a prototype for the following reasons, It's very flexible, it's local as they are based in Wellington and it has the ability to custom develop the admin area. The most important developments of this prototype were the ability to set custom usability rules when it came to adding new page templates. To create a page you had to start by telling the system the location where you want to place such page. For example, you wanted to create a student project. To be able to do this you had to specify the location in a drop-down box, then once selected all templates who are not part of projects will simply become unavailable or grey out in the admin area. This feature is to prevent the admin user to create a new item on the project location or vice versa. 


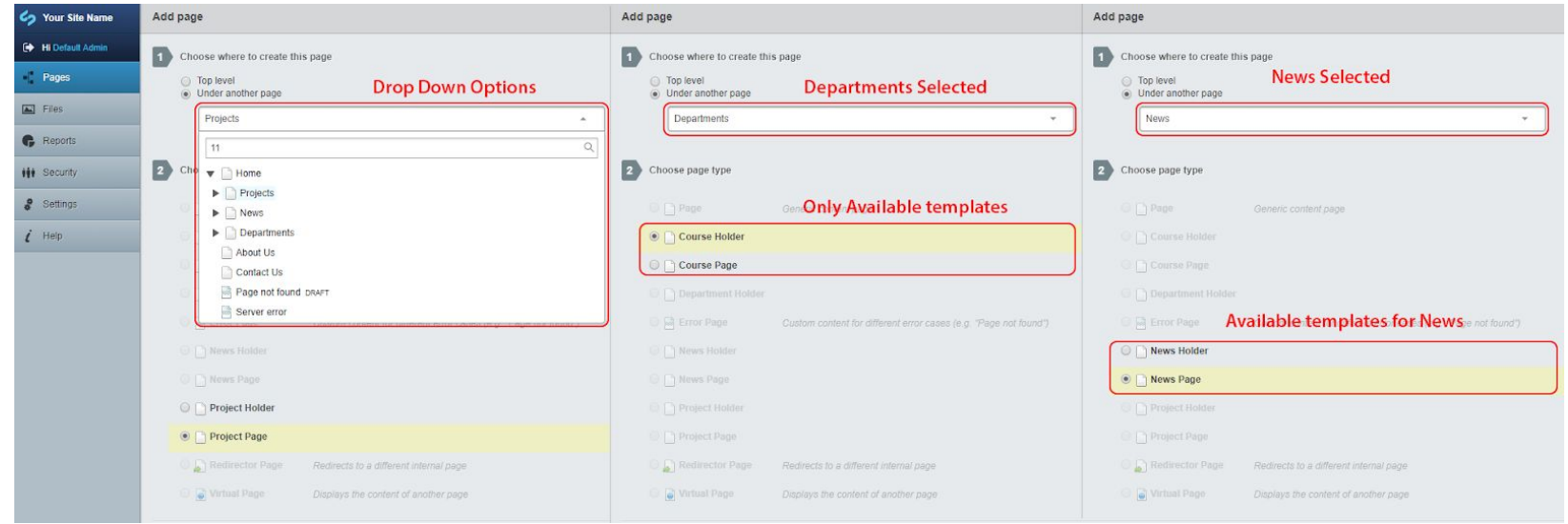

Figure 12. Usability design experiments in the SilverStripe prototype. Screenshot taken from local machine.

Apart from the usability rules for creating content, I developed several page templates for the admin area. Including templates for news, projects and courses. For these templates, I had to figure out how to create custom field types to be able to add custom fields for text entries and also for different types of formats such as dropdowns, dates, images and files. 


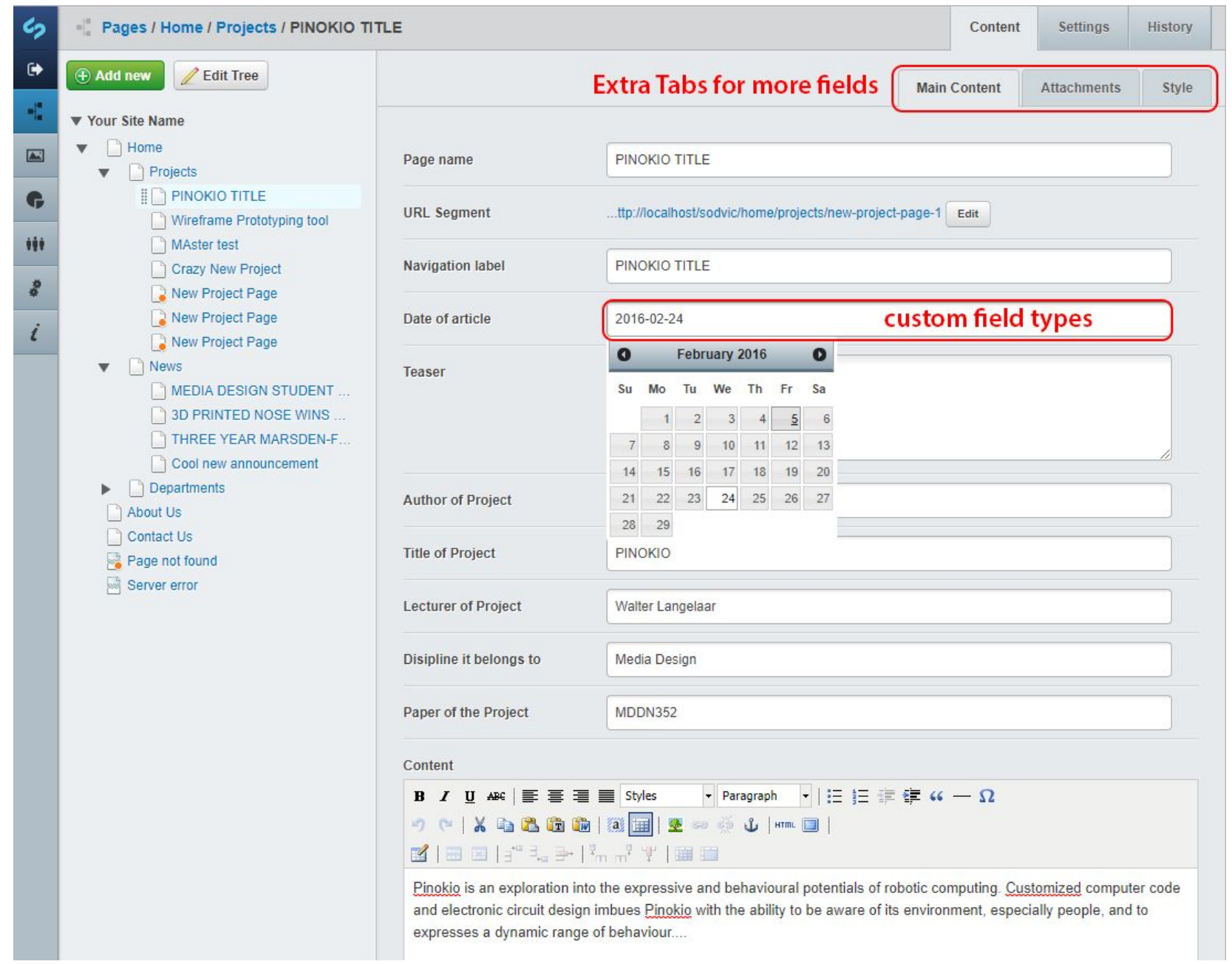

Figure 13. Usability design developments of the page editor and field types in the SilverStripe prototype. Screenshot taken from local machine. 


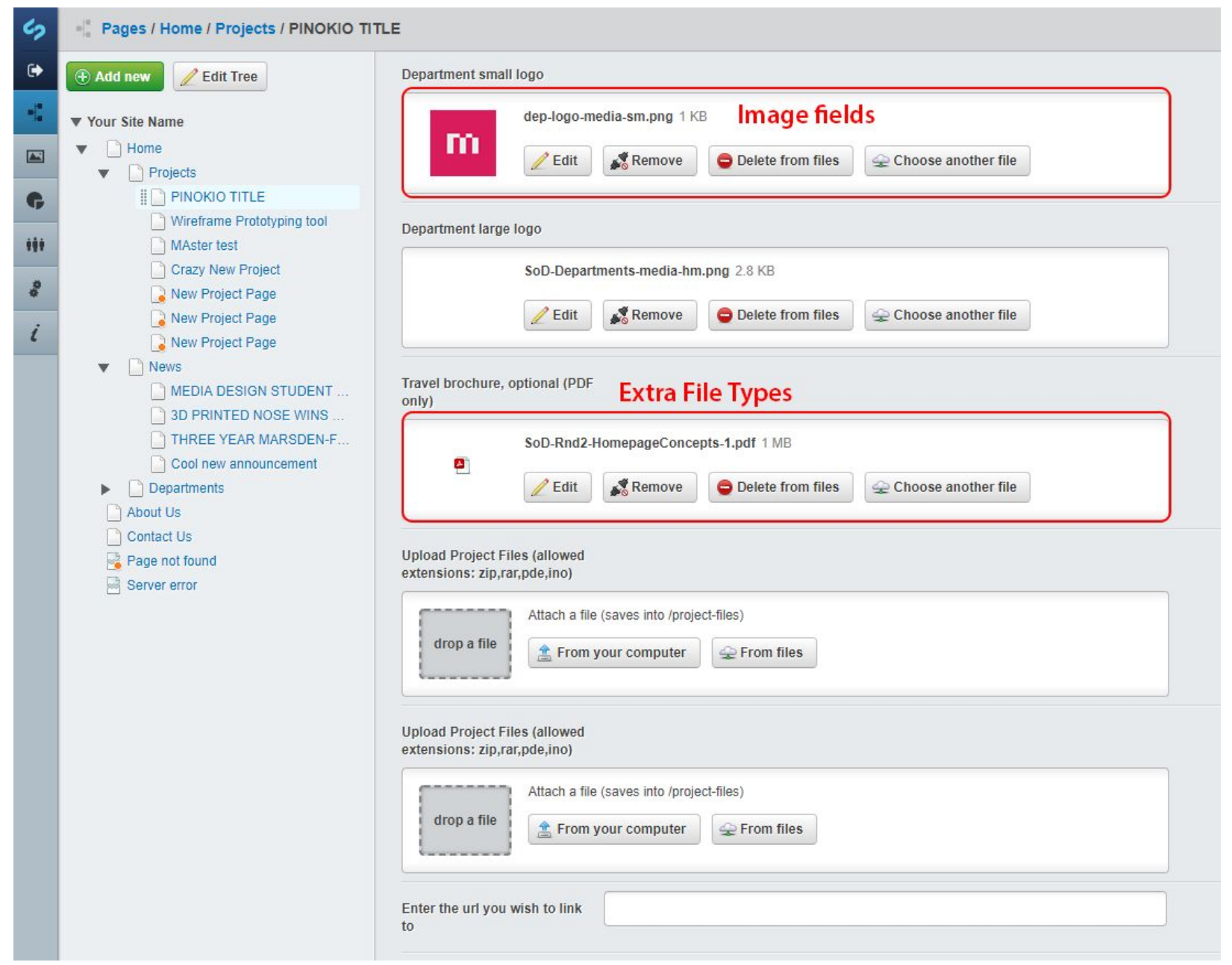

Figure 14. Permissions for field types of different file extensions in the SilverStripe prototype. Screenshot taken from local machine.

I made some important developments using SilverStripe CMS and it seemed like a promising platform to continue the developments. However, there were some complications that hampered the development which was due to the lack of documentation and user-generated content I could base myself on. Because of this, I decided to try and develop a new prototype using a different content management system. 


\section{Wordpress CMS prototype and analysis}

The move to the develop a prototype in WordPress was an easy decision, as WordPress boasts the largest CMS community on the internet. Because of this community, WordPress has a huge collection of development documentation such as tutorials and user-generated modules that are available to use and develop with.

When I think of a Content management system. I think of it as a book of forms. As each page is a different type of form that is asking for a specific type of data. Jarret \& Gaffney(2009) states that asking for data from people requires a commitment from them. Even if its tiny. To ensure getting the correct data from users I have to make sure that I know the user's relationship with the organization, understand the user's goals and to pick the right moment to ask a question.

There will only be two types of users that will have access to the admin area. The first will be a student user which will only be given to the representative of each course. This type of user will only have access to create student work and announcements for the course he/she represent. And the staff which will have access to most of the site including the same ability as the student but also having access to features such as creating and modifying courses, having the ability to upload content and files for each course.

\section{The Dashboard}


As mentioned previously WordPress biggest advantage is the ease of use but at the same time, it is a double-edged sword, as it makes some compromises in terms of complex functionality. But this lack of functionality only relates to the vanilla or out of the box state of a WordPress install. But the prototype in discussion was heavily customized to include many other features that by default is not available. Some of these features being the ability to modify the dashboard and add new custom post types.

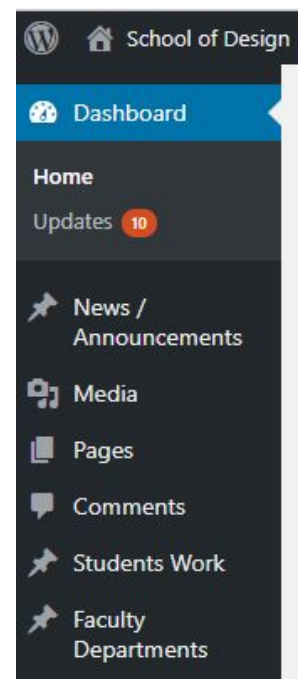

Figure 15. Dashboard of Wordpress prototype. Screenshot taken from local machine.

As shown in the image above, the dashboard contains a few different sections and each section is coded different to display a different template for the forms. These sections are :

- News and announcements

- Media - where all images and files are located and uploaded

- Pages - where the other pages will be located such as information about library, contact page, resources.

- Student work - All selected student projects will be stored to showcase in the website.

- Faculty - Departments and courses will be stored in a hierarchy format. 


\section{What is a post type?}

Post Types is a term used to refer to different types of content on a WordPress site. In 2003, WordPress was primarily launched as a blogging platform. Posts is a common blogging terminology that stuck with WordPress as it evolved into a robust content management system (CMS). When WordPress added different type of content, pages, they called it a different type of post hence post type. In the later versions, WordPress added the ability for developers to register their own custom post type. In all practical sense, post type is a content type. (WPbegginner, n.d.)

Vanilla WordPress comes with posts and pages as the initial post types, Usually, posts are reserved for news items and pages are used for the rest of pages like home, about us etc...

For a big community website like the one discussed in this project, having two post types is just not enough. With this in mind, I made two custom post types, one for departments and courses, and the other for student work.

\section{Page editor}

The out of the box state of Wordpress page editor is very simple in content. It only has a title input field and a WYSIWYG editor. In computing, a "WYSIWYG" editor is a system in which content (text and graphics) can be edited in a form closely resembling its appearance when printed or displayed as a finished product, such as a printed document, web page, or slide presentation. 
While having this kind of editor is fine for most small websites and blogs, this type of formatting won't be enough for this project. This website manages a large amount of data that needs to be organized and categorized. To solve this I developed a custom page editor for each post type. As you can see in figure (add figure numbers) The image to the left is the vanilla(define vanilla) page and to the right is the custom build page editor. In the custom version has many new types of input fields to properly organize data, data like author names, department and many more. Another thing that was added are the tabs to the editor that act as a navigation, to be able to quickly go to a specific section rather than scroll down on a long form.
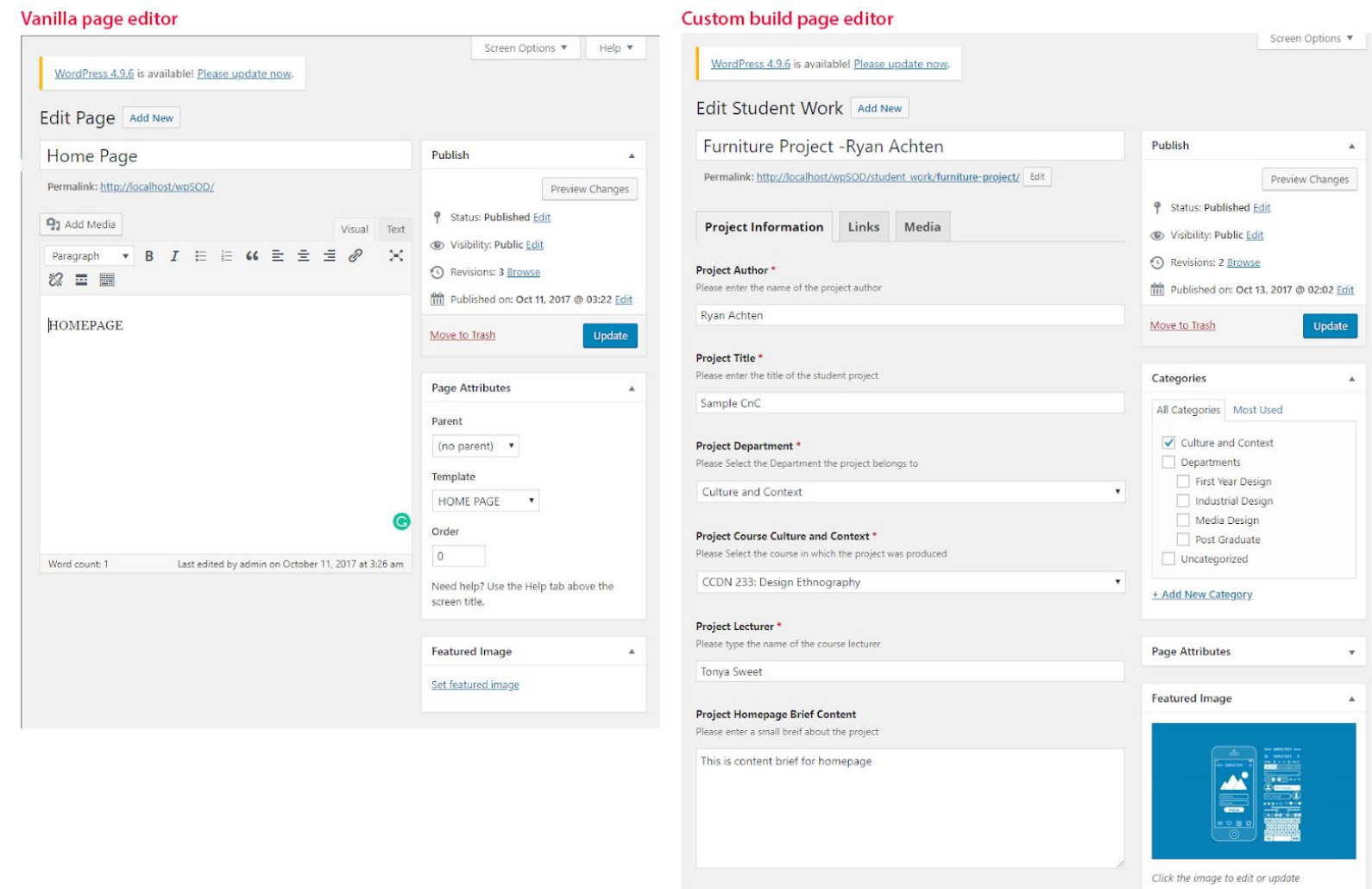

Figure 16. Side by side of the vanilla version and wordpress prototype page editor. Screenshot taken from local machine.

Having these new features aided in the organization of data but it also made a new challenge. This challenge is the addition of many smaller tasks in which the user can 
get confused. To solve this. the custom built prototype has a label field that contains instructions for each input field and the best thing about it is that these instructions can also be edited in the CMS without needing to code them.

This ability to add instructions gave this system one of the biggest boosts to the usability which is "language". In the UX 2017 NZ conference, Kah Chan makes an interesting talk about the importance of Crafting Language in UX (named "Why we need to write well for other people'). Kah spoke how integral language is to the user experience and that our words should convey empathy, simplicity and clarity. (Chan, 2017).

\section{Conditional statements}

In computer science, conditional statements are features of a programming language, which perform different computations or actions depending on whether a programmer-specified condition evaluates to true or false.

As mentioned previously this website will house a large amount of data, some of this data are lists of courses and there is a large amount of them. This large amount of courses can generate a bit of a usability challenge as I don't want the user to be overwhelmed by a large drop-down field. To avoid this I had the idea to add conditional statements. These conditional statements were added to the forms to create more complex functionality, for example in the figure(department/course) there are two input fields tied together with a conditional statement. So Instead of having a large drop-down of courses, it has been simplified to first choosing the department and when this action is done, the second field refreshes and will only list the courses that belong to the selected department, making this dropdown list much shorter and easier to select. 


Project Department *
Please Select the Department the project belongs to
Media Design
Project Course Media Design *
Please Select the course that the project belongs to
MDDN 201: Internet Design
MDDN 201: Internet Design
MDDN 211: Digital Video Creation
MDDN 241: 3D Modelling and Animation II
MDDN 242: Creative Coding II
MDDN 243: Introduction to Computer Game Design
MDDN 251: Physical Computing
MDDN 311: Postproduction and Special Effects
MDDN 314: Audio-Visual Space
MDDN 342: Creative Coding III
MDDN 343: Advanced Computer Game Design
MDDN 351: Wearable Technology
MDDN 352: Mobile Media
MDDN 381: Independent Study

Figure 17. Conditional statements in the admin area. Screenshot taken from local machine.

However this is not the only use for conditional statements, as this development was also applied to the creation of departments and courses, the Challenge here was that both departments and courses share the same post type but they are of a different page design. But in WordPress, you can only have one page template per post type. To fix this I developed two separate conditional statements and tied them together; one for WordPress admin area and another for frontend section. So the website will choose what page template to render whether it is a department or a course. The figure (figure) is an example of this in action. The left side is the first action that the user will need to take. This action is to choose whether you are creating a new department or a new course. The form will automatically refresh and add the tabs containing all organised input fields depending on the user decision. 


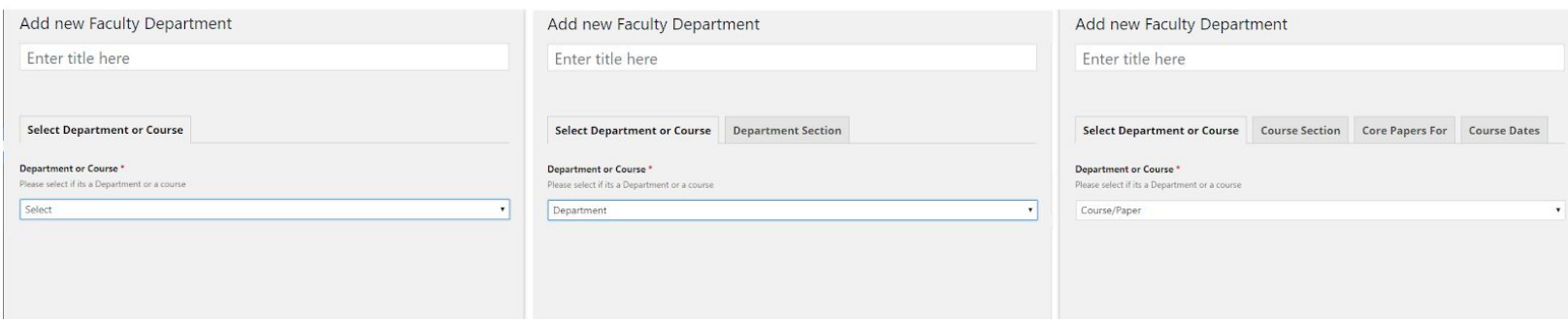

Figure 14. Conditional statements to create either a department or a course. Screenshot taken from local machine.

\section{Reflection}

While I consider myself a generalist designer, my focus has always been web design and development. As a designer I try to find a solution to a problem but at the same time I like to get my hands dirty in the development of such solutions. I have worked on developing websites with content management systems in the past. But during these jobs I took for granted the consideration of the admin user: Their experience through the admin area, the relationship with the frontend(findway to format) and the collaboration that can happen inside it. I always just treated it as just a database.

This research helped me realise the amount of consideration we can take as designers when developing a systems that will be used by the non tech savvy user. Considerations need to be made such as the ease of use of the admin area, the presentation of the data in the frontend and the impact that online collaboration has in a community website like the academic community of the School of Design discussed in here. 


\section{Online collaboration}

The research in online collaboration helped me understand the social aspects of an online collaborative design community and what constitutes an online collaboration. From what I learn I can state that what makes an online collaboration are the following points

1. Rules for participation

2. Organizational structure and leadership

3. Established guidelines for attribution

4. Clear goals

5. Tools

All of these points were applied throughout the design and development of the School of Design website. The rules for participation relates to the code of conduct that the user needs to follow. The organizational structure relates to the user roles such as student, teacher and administrator. The guidelines for attribution relates to the user permission within this system so for example, students are only able to add and edit student work and course news related with each student representative and staff are able to do the same as a student but also the ability to add courses. The clear goal of this website is the maintenance and input of new exemplary student work to showcase and new information relating to courses. Finally, the tools for collaboration is the custom build content management system itself. 


\section{Usability design}

Not only did I used to take for granted the usability design within content management systems but also the usability of the front-end when it came to designing websites with a large amount of content. Previously I used to design websites concentrating more on aesthetics rather than its functionality. As discussed in this research, users are always in a hurry, they want to get to their destination quickly. This gave way for a navigation design and the display of content within the School of Design website. These features being the filtering navigation and the tile format for displaying content within the website.

These considerations were applied to this project within the experimentations of the iterative approach to this thesis. With every design iteration, I applied new design choices and I kept learning along the way. Until I arrived at a design I was happy with and that resolved some of the problems I had. The same happened with the development of the software itself. The testing of each CMS prior to the development helped me find their strengths and weaknesses and with these findings, I was able to choose WordPress and SilverStripe to develop the prototypes. For every prototype of a CMS I experimented with, some new possibilities came to light and as well as challenges. For example, while on the development of the SilverStripe prototype helped me learn new features I could do for the usability of the admin area. The challenges helped me make the decision to halt the development of the SilverStripe prototype and move into developing one for WordPress. as discussed previously these challenges being the lack of documentation and low user development community. 


\section{The output}

The practical output of this research is separated in two sections, a frontend design theme and the backend development of the admin area. Both combined form the website for the Victoria University School of Design.

\section{Front-end design}

PDF can be downloaded here - https://mega.nz/\#!G01SzYra
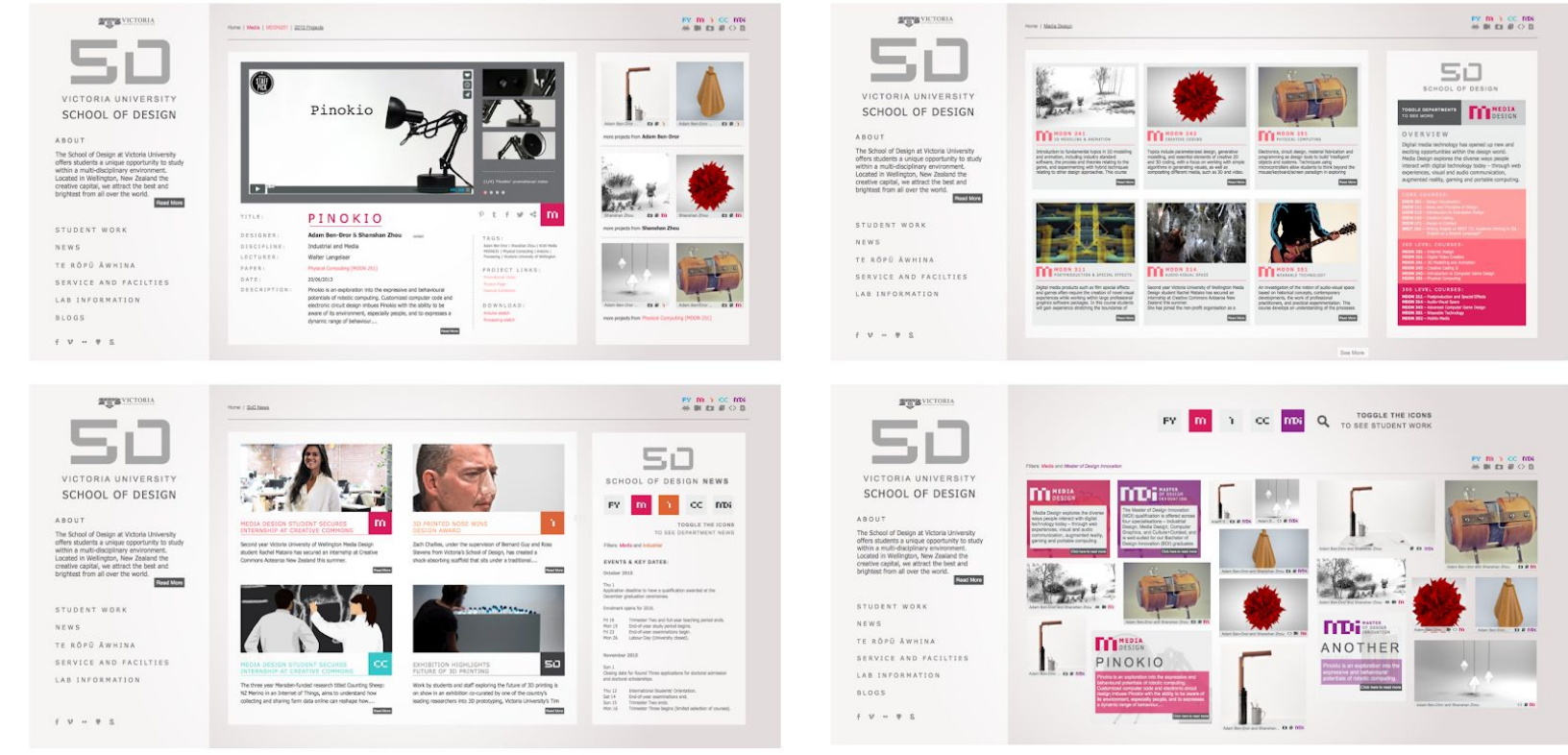

Theme is built using HTML, CSS, JavaScript, jQuery, AJAX and PHP

Links to final design and theme files are in the web version of the thesis. 


\section{The CMS}

The final prototype was developed using Wordpress as a CMS. A custom built system that improves upon the standard version.

All content in the site is all sample content and images used are all mine and from some friends which I asked their permission

Link to the website : www.sebseb.co/thesis/

: www.sebseb.co 


\section{Conclusion}

This study focused on creating a user-friendly experience for a sustainable collaborative academic community website showcasing different works. The "users" in this case includes frontend users such as prospective students, current students and their friends and family, as well as administration users such as student reps, lecturers, course admin and tutors.

As discussed earlier, content management systems in their vanilla state come with limited functionality and it is left for the developer to improve upon. However, some of these don't have the ability to let you modify specific parts of the admin area to improve usability. Therefore only some of these CMSs were selected to develop prototypes in it. These prototypes took me to the final iteration of a heavily custom built WordPress website and a theme for it. (The theme for a CMS is a collection of code files and (usually) images that determine how a CMS website looks).

My output fulfils what I was looking to design as set out in my brief. I was able to improve the usability of the admin area and tailored the design of the frontend website for an easier to navigate and easy access to content. However, it is not without downsides. Some of this downsides are the way this final software was built. As it was developed using an existing content management system. In this case WordPress, it relies on a combination of plugins and my own development that are not part of the vanilla state. Because of this, replicating this development to a new project will not be as simple as a copy and paste as it has many dependencies in which it relies upon.

With the rapid advancements of web technologies and with the ever-increasing updates of this content management systems. There could be a point in which these usability improvements are added to the out of the box state of WordPress or other CMSs. This would improve the user experience and enable the different user roles to properly collaborate with less reliance on a developer to custom build the CMS. 


\section{References}

Collaborative Future.(2010). Collaborative Futures retrieved from

http://write.flossmanuals.net/collaborativefutures/anonymous/

Fuller, M. (2003). Behind the blip: essays on the culture of software. Canada:

Autonomedia.

Cooper, A. (1999). The Inmates are Running the Asylum. Indianapolis: Sams Publishing

Hemetsberger, A., Reinhardt, C. (2009) Collective Development in Open-Source

Communities: An Activity Theoretical Perspective on Successful Online Collaboration.

Organization Studies, 30(9), 987-1008.

Engeström, Y. (2004). New forms of learning in co-configuration work'. Journal of Workplace Learning, 16(2), 11-21.

Zablith, F., Faraj, S., \& Azad, B. (2016) Organizational knowledge generation: lessons from online communities, Business Process Management Journal, 22, 33-55.

Miaskiewicz, T., Kozar, K. (2011). Personas and User-centered Design: How can Personas Benefit Product Design Processes?, Design Studies, 32(5), 417-430.

Norman, D. (2013). The design of everyday things (4th ed.). (n.p)

Carroll, J., Zahirovic, A., Lowgreen, J., Hassenzahl, M., Erickson, T. (n.d.). The Encyclopedia of Human-Computer Interaction, 2nd Ed. retrieved from https://www.interaction-design.org/literature/book/the-encyclopedia-of-human-compu ter-interaction-2nd-ed/ 
Krug, S. (2014). Don't make me think (3rd ed.). Berkeley, California,: New Riders

Lury, C., Wakeford, N. (2012). Inventive methods: the happening of the social. London: Routledge

Joomla.org. (n.d). Joomla. retrieved from https://www.joomla.org/

Wordpress.org. (n.d). Wordpress. retrieved from https://www.joomla.org/

Silverstripe limited. (n.d). SilverStripe. Retrieved from http://www.silverstripe.org/ Gillan,D, Holden,J, Adam,S, Rudisill,M, Magee,L; How should Fitts' Law be applied to human-computer interaction?, Interacting with Computers, Volume 4, Issue 3, 1 December 1992, Pages 289-290, https://doi-org.helicon.vuw.ac.nz/10.1016/0953-5438(92)90018-B

Chan,K (2017, October). The importance of crafting language. UX New Zealand. Wellington. Retrieved from http://www.uxnewzealand.com/speakers/kah-chan/

Gaffney, G., Jarret,C. (2009). Forms that work (1st ed). Morgan Kaufmann

Carroll, Zahirovic, Lowgreen, Hassenzahl, Erickson. Human Computer Interaction [Online image]. Retrieved from https://www.interaction-design.org/literature/book/the-encyclopedia-of-human-compu ter-interaction-2nd-ed/human-computer-interaction-brief-intro

Carroll, Zahirovic, Lowgreen, Hassenzahl, Erickson. User Experience/Usability [Online image]. Retrieved from 
https://www.interaction-design.org/literature/book/the-encyclopedia-of-human-compu ter-interaction-2nd-ed/human-computer-interaction-brief-intro 